\title{
Mesoscale Nonhydrostatic and Hydrostatic Pressure Gradient Forces-Theory
}

\author{
G. A. DAlu AND M. BALdi \\ Department of Atmospheric Science, Colorado State University, Fort Collins, Colorado, and Institute of Atmospheric Science and \\ Climatology, ISAC-CNR, Rome, Italy \\ R. A. Pielke SR. AND G. LeONCini \\ Department of Atmospheric Science, Colorado State University, Fort Collins, Colorado
}

(Manuscript received 13 February 2002, in final form 28 February 2003)

\begin{abstract}
A theory is presented for the evaluation of the different terms of the pressure gradient force, when mesoscale flow is driven by a sensible heat source in the planetary boundary layer (PBL), or by an elevated confined heat source, such as the release of the latent heat of condensation in a cloud. The nonlinear and linear, and the nonhydrostatic and the hydrostatic pressure gradient contributions are evaluated. The validity of the different approximations is discussed as a function of time and space scales. In addition, the validity of this approach is explored as a function of atmospheric environmental parameters, such as static stability, large-scale flow, and dissipation.

By accessing the relative importance of each contribution, specific solution techniques for mesoscale atmospheric flows can be adopted. For example, when the linear contributions dominate, an exact analytic model could be used, rather than relying on numerical approximation solution techniques. When the hydrostatic contribution dominates, the spatial variation of the vertical temperature profile can be used to uniquely define the horizontal pressure gradient force.
\end{abstract}

\section{Introduction}

Hydrostatic and linear approximations to the equations that describe atmospheric flow are frequently made in order to simplify their integration in time. Pielke (2002) describes the use and application of these two solution techniques. The hydrostatic approximation is useful since, when it accurately describes atmospheric flow, the pressure field is directly diagnosed from the instantaneous temperature field. The use of the linear approximation permits analytically exact solutions. Moreover, the linear approximation is a realistic representation of atmospheric flow when nearly linear forcing occurs (e.g., the horizontal pressure gradient force, $\nabla_{\mathrm{H}} p / \rho \cong \nabla_{\mathrm{H}} p / \rho_{0}$ where the density $\rho_{0}$ is only a prescribed function of $z$ ).

Nonhydrostatic versus hydrostatic flow has been extensively studied theoretically and numerically. In geophysics, there are many flows where these differences are relevant. One obvious application is the lee wave, where the aspect ratio is of order one and the time scale is strictly related to the Brunt-Väisälä frequency (Sun 1984; Smith 1989; Rõõm and Männik 2000; Thunis and

Corresponding author address: Dr. R. A. Pielke Sr., Dept. of Atmospheric Science, Colorado State University, Fort Collins, CO 80523. E-mail: pielke@atmos.colostate.edu
Clappier 2000). There are studies of the unstable convective boundary layer and thermocline, which show that nonhydrostatic terms are important where thermals are present (Seman 1994; Molemaker and Dijkstra 2000). Nonhydrostatic effects are, of course, important in cloud dynamics (Cotton and Anthes 1989).

Song et al. (1985) proposed a procedure to decompose a set of atmospheric equations into hydrostatic and nonhydrostatic components, while Weidman and Pielke (1983) described a technique to segment linear and nonlinear terms.

Using the modeling framework represented in Dalu et al. $(1996,2000)$, we apply this methodology in order to develop an improved understanding of the role of hydrostatic and nonhydrostatic, and linear and nonlinear contributions to the pressure gradient force associated with thermally forced mesoscale flows. Our study, which is fully analytical, is focused on the quantitative evaluation of the pressure terms as a function of the space-time scale and of the atmospheric stability, dissipation and diffusion, and large-scale flow.

The pressure perturbation directly induced by a diabatic source forces a flow which, through the convergence-divergence of mass and convergence-divergence of buoyancy within a column of stratified air, linearly perturbs the pressure field. In addition there is a pressure 
perturbation, which results from the nonlinear convergence-divergence of buoyancy and a nonlinear dynamics pressure term.

We examine the relative importance of these terms as a function of the time-space scales and of the environmental parameters. In addition we suggest a parameterization (a correction term) that reintroduces most of the nonhydrostatic effect in a hydrostatic flow.

Our study is presently two-dimensional. A fully 3D approach would allow the analysis of other important phenomena as, for instance, in Rotunno and Klemp (1982), who using a simplified nonlinear model, complemented by a semiquantitative linear analysis, studied the asymmetric growth of a low-high dipole induced by the veering of the vertical shear of the large-scale flow, which favors the cyclonic vortex and inhibits the anticyclonic vortex. Three-dimensionality and veering of the vertical shear are essential for the asymmetric development of the vortex pair.

\section{The equations: Nonhydrostatic, hydrostatic, linear, and nonlinear forms}

The governing equations for the dynamics are reported in the appendix; here, we present the equations as derived from the Navier-Stokes equations. In these equations $u, v$, and $w$ are the momentum components, $U$ is the large-scale flow intensity, $b$ is the buoyancy force, $Q$ is the diabatic buoyancy source related to the sensible heat flux in the convective boundary layer (CBL) or the latent heat released in a cloud, and $\phi$ is the geopotential as in Rotunno (1983). The horizontal pressure force is related to the horizontal gradient of the geopotential by

$$
g \nabla_{p} z=\alpha \nabla_{z} p=\nabla_{p} \phi,
$$

where $\alpha$ is the specific volume of the air. In the following derivation the gradient of $\phi$ is used to represent the pressure gradient force.

\section{a. Environmental parameters}

We use the following values for the Brunt-Väisälä frequency, Coriolis parameter, and diffusion coefficient.

$$
\begin{aligned}
\Theta_{z} & =1 \text { to } 5\left(\mathrm{~K} \mathrm{~km}^{-1}\right) ; \quad b=g \frac{\theta}{\Theta} \\
N & =\left(g \frac{\Theta_{z}}{\Theta}\right)^{1 / 2}=3 \times 10^{-3} \text { to } 2 \times 10^{-2}\left(\mathrm{~s}^{-1}\right) ; \\
K & =10 \text { to } 100\left(\mathrm{~m}^{2} \mathrm{~s}^{-1}\right) ; \\
\omega_{0} & =1 \text { to } 100(2 \pi / \text { day }) \\
f & =2 \omega_{E} \sin (\text { lat })=10^{-4}\left(\mathrm{~s}^{-1}\right) \\
\lambda & =1 \text { to } 100\left(\text { day }^{-1}\right) \\
U & =0 \text { to } 10\left(\mathrm{~m} \mathrm{~s}^{-1}\right) ;
\end{aligned}
$$

where $\omega_{0}$ is the pulsation of the forcing, $\omega_{E}=(2 \pi /$ day $)$ is the angular velocity of the earth, $\theta$ is the potential temperature perturbation, $\Theta$ is the environment potential temperature, and $\Theta_{z}$ is its vertical gradient. The lifetime of the mesoscale flow is $\lambda^{-1} ; \lambda$ is the Rayleigh friction coefficient, that is, a bulk dissipation that represents frictional losses at low wavenumbers; and $K$ is the diffusion coefficient that accounts for losses at high wavenumbers. In nature during the day, within the convective atmospheric boundary layer the diffusion process is very active and the diffusion coefficient $K$ is very high. In the free atmosphere and during the night, the diffusion processes are weak and the diffusion coefficient $K$ is low (Stull 1988). In the results shown hereafter, $K$ is constant.

\section{b. Mesoscale forcing}

The mesoscale flow is driven by the buoyancy gradient generated by the diabatic source $Q$ in Eq. (A4):

$$
Q=Q_{0} q(t) r(x, z)
$$

where $q(t)$ is its time behavior and $r(x, z)$ its spatial distribution. The diabatic perturbation of the buoyancy $B$ directly induced by the heat source $Q$ is

$$
B=Q_{0} r(x, z) \int_{0}^{t} q\left(t^{\prime}\right) d t^{\prime} .
$$

\section{c. The linear equations}

\section{1) THE LINEAR OPERATOR AND ITS FOURIER TRANSFORM}

We define the linear operator $\mathcal{L}$, and formally its inverse $\mathcal{L}^{-1}$ and its square $\mathcal{L}^{2}$ :

$$
\begin{aligned}
\mathcal{L} & \equiv\left(\frac{\partial}{\partial t}+\lambda+U \frac{\partial}{\partial x}-K \nabla^{2}\right) ; \\
\mathcal{L}^{-1} \mathcal{L} f(x, z, t) & =f(x, z, t) ; \\
\mathcal{L} \mathcal{L} f(x, z, t) & =\mathcal{L}^{2} f(x, z, t) .
\end{aligned}
$$

We define the Laplacian operator $\nabla^{2}$ and formally the inverse Laplacian operator $\nabla^{-2}$ :

$\nabla^{2} \equiv \frac{\partial^{2}}{\partial x^{2}}+\frac{\partial^{2}}{\partial z^{2}} ; \quad \nabla^{-2} \nabla^{2} f(x, z, t)=f(x, z, t)$.

We define the Fourier transform $\mathcal{F}\{f(x, z, t)\}=\tilde{f}(k, \nu$, $\omega)$, and its inverse $\mathcal{F}^{-1}\{\tilde{f}(k, \nu, \omega)\}=f(x, z, t)$. Then the Fourier transforms of the operator $\tilde{\mathcal{L}}$, its inverse $\tilde{\mathcal{L}}^{-1}$, and its square $\tilde{\mathcal{L}}^{2}$ are

$$
\begin{aligned}
\tilde{\mathcal{L}} & \equiv\left[\lambda+K\left(k^{2}+\nu^{2}\right)\right]+i(\omega+k U)=D+i \Omega ; \\
\tilde{\mathcal{L}}^{-1} & =\frac{D-i \Omega}{D^{2}+\Omega^{2}} ; \quad \tilde{\mathcal{L}}^{2}=D^{2}-\Omega^{2}+2 i \Omega D \\
D & =\lambda+K\left(k^{2}+\nu^{2}\right) ; \quad \Omega=(\omega+k U) .
\end{aligned}
$$


In Eq. (8), $\tilde{\mathcal{L}}$ is a complex pulsation where the pulsation of $\omega$ is corrected by the large-scale advection, dissipation, and diffusion. The Fourier transforms of the Laplacian operator $\tilde{\nabla}^{2}$ and of its inverse $\tilde{\nabla}^{-2}$ are

$$
\tilde{\nabla}^{2} \equiv-\left(k^{2}+\nu^{2}\right) ; \quad \tilde{\nabla}^{-2}=-\frac{1}{\left(k^{2}+\nu^{2}\right)} .
$$

If $f(x, z, t)$ is the dynamical response to a periodic forcing $Q(x, z, t)$ through the operator $\mathcal{L}$,

$$
\begin{aligned}
\mathcal{L} f(x, z, t) & =Q(x, z, t) \\
& =Q_{0} \sin (\omega t+k x+\nu z) .
\end{aligned}
$$

Then, in the presence of large-scale advection $U \neq 0$, but in the absence of dissipation and diffusion $D=0$, there is an attenuation of the response $f(x, z, t)$ :

$$
\begin{aligned}
f(x, z, t)= & -\frac{Q_{0} \cos (\omega t+k x+\nu z)}{(\omega+k U)} \\
= & \frac{Q_{0} \sin \left(\omega t+k x+\nu z-\frac{\pi}{2}\right)}{(\omega+k U)} .
\end{aligned}
$$

While in the presence of large-scale advection $U \neq 0$ and in the presence of dissipation and diffusion $D \neq 0$ there is an attenuation and a phase shift of the response:

$$
f(x, z, t)=\frac{D Q_{0} \sin (\omega t+k x+\nu z)-\Omega Q_{0} \cos (\omega t+k x+\nu z)}{D^{2}+\Omega^{2}} .
$$

\section{2) LIFETIME AND LAPLACE TRANSFORM}

If $f(x, z, t)$ is the dynamical response to an impulsive forcing in the presence of dissipation and diffusion and in the absence of large-scale flow, $U=0$ and $D \neq 0$,

$$
\begin{aligned}
\mathcal{L} f(x, z, t) & =Q(x, z, t) \\
& =Q_{0} \sin (k x+\nu z)[\operatorname{He}(\mathrm{t})-\operatorname{He}(t-T)] .
\end{aligned}
$$

The Heaviside step function, which is denoted by He, is $[\mathrm{He}(t)-\mathrm{He}(t-T)]=1$ for $0 \leq t<T$, and $T$ is the duration of the impulse. If $s$ is the Laplace transform of the time and $\hat{f}(x, z, s)$ is Laplace transform of $f(x$, $z, t)$, then

$(s+D) \hat{f}(x, z, s)=Q_{0} \sin (k x+\nu z) \frac{1}{2}[1-\exp (-T s)]$

$$
\begin{aligned}
f(x, z, t) & \\
= & \tau Q_{0} \sin (k x+\nu z) \\
\quad \times & \left\{\operatorname{He}(t)\left[1-\exp \left(-\frac{t}{\tau}\right)\right]\right. \\
& \left.\quad-\operatorname{He}(t-T)\left[1-\exp \left(-\frac{t-T}{\tau}\right)\right]\right\},
\end{aligned}
$$

when $t>T$ then

$$
\begin{aligned}
f(x, z, t)= & \tau Q_{0} \sin (k x+\nu z) \\
& \times\left[\exp \left(-\frac{t-T}{\tau}\right)-\exp \left(-\frac{t}{\tau}\right)\right], \\
\tau= & \frac{1}{\operatorname{Re}\{\tilde{L}\}}=\frac{1}{D}=\frac{1}{\lambda+K\left(k^{2}+\nu^{2}\right)} .
\end{aligned}
$$

When the forcing ends, the flow decays with a lifetime $\tau$. This result can be generalized using the shifting theorem in the Laplace transform and the Faltung theorem in the inverse Laplace transform (LaPage 1961).

\section{(i) Linear equations}

The linear and nonhydrostatic form of the equations are

$$
\begin{aligned}
& \mathcal{L} u^{L_{\mathrm{NH}}}-f \boldsymbol{v}^{L_{\mathrm{NH}}}+\phi_{x}^{L_{\mathrm{NH}}}=0 ; \\
& \mathcal{L} \boldsymbol{v}^{L_{\mathrm{NH}}}+f \boldsymbol{u}^{L_{\mathrm{NH}}}=0 ; \\
& \mathcal{L} w^{L_{\mathrm{NH}}}+\phi_{z}^{L_{\mathrm{NH}}}=b^{L_{\mathrm{NH}}} \\
& \mathcal{L} b^{L_{\mathrm{NH}}}+N^{2} w^{L_{\mathrm{NH}}}=Q ; \\
& u_{x}^{L_{\mathrm{NH}}}+w_{z}^{L_{\mathrm{NH}}}=0 .
\end{aligned}
$$

(ii) The linear-nonhydrostatic geopotential equation

To derive the linear geopotential from Eq. (18), we differentiate the first momentum equation by $x$ and multiply by $\mathcal{L}$, add the second momentum equation differentiated by $x$ and multiplied by $f$; add the third momentum equation differentiated by $z$ and multiplied by $\mathcal{L}$, then add the buoyancy equation differentiated by $z$ yielding

$$
\mathcal{L} \nabla^{2} \phi^{L_{\mathrm{NH}}}=\frac{\partial Q}{\partial z}-\left[f^{2} \frac{\partial u^{L_{\mathrm{NH}}}}{\partial x}+N^{2} \frac{\partial w^{L_{\mathrm{NH}}}}{\partial z}\right],
$$

which, using continuity [Eq. (A4)], becomes

$$
\mathcal{L} \nabla^{2} \phi^{L_{\mathrm{NH}}}=\left[\partial_{z} Q+\left(N^{2}-f^{2}\right) \partial_{x} u^{L_{\mathrm{NH}}}\right] .
$$

In the previous equation, the first term on the right side is the contribution to the geopotential, directly induced by the buoyancy source through its vertical divergence, 
$\phi_{Q}$ in Eq. (20). The second term on the right-hand side is the contribution to the geopotential due to the divergence-convergence of mass within the same column of stratified air, $\phi_{D}^{L_{\mathrm{NH}}}$ in Eq. (21). These two terms are linear

$$
\begin{aligned}
\mathcal{L} \nabla^{2} \phi_{Q} & =\partial_{z} Q \\
\mathcal{L} \nabla^{2} \phi_{D}^{L_{\mathrm{NH}}} & =\left(N^{2}-f^{2}\right) \partial_{x} u^{L_{\mathrm{NH}}} .
\end{aligned}
$$

In addition, we evaluate the geopotential contribution $\phi_{B}^{L_{\mathrm{NH}}}$ due to the convergence-divergence of the diabatically modified air [Eqs. (5) and (A27)] for comparison with $\phi_{Q}$ and $\phi_{D}^{L_{\mathrm{NH}}}$ :

$$
\mathcal{L} \nabla^{2} \phi_{B}^{L_{\mathrm{NH}}}=-\partial_{z}\left(u^{L_{\mathrm{NH}}} \partial_{x} B+w^{L_{\mathrm{NH}}} \partial_{z} B\right) .
$$

Note that $\phi_{B}^{L_{\mathrm{NH}}}$ is also linear. Therefore, there are three linear contributions to the geopotential. The dynamics are driven by the gradient of $\phi_{Q}$, which is the component of the geopotential directly induced by the heat source $Q$. Here $\phi_{D}^{L_{N H}}$ is the component of the geopotential induced by the divergence-convergence of mass within the same column of air and is directly linked to the strength of the stratification of the environment $N^{2}$. Additionally $\phi_{B}^{L_{\mathrm{NH}}}$ is the component of the geopotential induced by the divergence-convergence of the diabatically modified air within the same column of air, which is a function of the strength and shape of the diabatic source that modifies the environment stability, and of the strength of the dynamical advection. The total linear nonhydrostatic geopotential $\nabla^{2} \phi_{G}^{L_{\mathrm{NH}}}$ is

$$
\nabla^{2} \phi_{G}^{L_{\mathrm{NH}}}=\nabla^{2} \phi_{Q}+\nabla^{2} \phi_{B}^{L_{\mathrm{NH}}}+\nabla^{2} \phi_{D}^{L_{\mathrm{NH}}} .
$$

\section{(iii) The linear-hydrostatic geopotential equation}

The hydrostatic and linear approximation of the geopotential equation is derived neglecting the vertical acceleration term, $\mathcal{L} w^{L_{\mathrm{NH}}}$, in the third momentum equation in Eq. (18), which then reduces to the hydrostatic pressure equation: $\phi_{z}^{L_{\mathrm{NH}}}=b^{L_{\mathrm{NH}}}$. We differentiate the first momentum equation by $x$ and multiply by $\mathcal{L}$, add the second momentum equation differentiated by $x$ and multiplied by $f$; add the hydrostatic pressure equation differentiated by $z$ and multiplied by $\mathcal{L}$, yielding

$\mathcal{L} \nabla^{2} \phi^{L_{H}}=\frac{\partial Q}{\partial z}-\left[f^{2} \frac{\partial u^{L_{H}}}{\partial x}+N^{2} \frac{\partial w^{L_{H}}}{\partial z}\right]-\mathcal{L}^{2} \frac{\partial u^{L_{H}}}{\partial x}$.

The hydrostatic geopotential equation has an extra divergence term, $-\mathcal{L} \partial_{x} u^{L_{H}}$, due to the broken symmetry between the first and the third momentum equations because of the hydrostatic approximation. In hydrostatic approximation, the component of the geopotential due to the divergence-convergence of the diabatically modified air is

$$
\mathcal{L} \nabla^{2} \phi_{B}^{L_{H}}=-\partial_{z}\left(u^{L_{H}} \partial_{x} B+w^{L_{H}} \partial_{z} B\right),
$$

The total linear hydrostatic geopotential $\nabla^{2} \phi_{G}^{L_{H}}$ is

$$
\nabla^{2} \phi_{G}^{L_{H}}=\nabla^{2} \phi_{Q}+\nabla^{2} \phi_{B}^{L_{H}}+\nabla^{2} \phi_{D}^{L_{H}}-\mathcal{L} \partial_{x} u^{L_{H}},(26)
$$

(iv) The nonhydrostatic contribution to the geopotential

We define $\delta \phi^{\mathrm{NH}}=\left[\phi^{L_{\mathrm{NH}}}-\phi^{L_{H}}\right]$ as the nonhydrostatic contribution to the geopotential, that is, the difference between the nonhydrostatic and the hydrostatic geopotential:

$$
\begin{aligned}
\mathcal{L} \nabla^{2} \delta \phi^{\mathrm{NH}}= & \mathcal{L} \nabla^{2}\left[\phi^{L_{\mathrm{NH}}}-\phi^{L_{H}}\right] \\
= & \left(N^{2}-f^{2}\right) \partial_{x}\left(u^{L_{\mathrm{NH}}}-u^{L_{H}}\right) \\
& -\partial_{z}\left[\left(u^{L_{\mathrm{NH}}}-u^{L_{H}}\right) \partial_{x} B-\left(w^{L_{\mathrm{NH}}}-w^{L_{H}}\right) \partial_{z} B\right] \\
& +\mathcal{L}^{2} \partial_{x} u^{L_{H}} .
\end{aligned}
$$

This Poisson equation for $\delta \phi^{\mathrm{NH}}$ contains hydrostatic and nonhydrostatic dynamical variables in its nonhomogeneous term.

\section{(v) The nonhydrostatic residual}

When the intensity of the nonhydrostatic flow does not differ too much from the intensity of hydrostatic flow,

$$
\left|\frac{u^{L_{\mathrm{NH}}}-u^{L_{H}}}{u^{L_{\mathrm{NH}}}}\right| \ll 1 \text { and } \quad\left|\frac{w^{L_{\mathrm{NH}}}-w^{L_{H}}}{w^{L_{\mathrm{NH}}}}\right| \ll 1,
$$

Eq. (27) can be approximated by Eq. (28):

$$
\nabla^{2} \delta \phi^{\mathrm{NH}} \approx \nabla^{2} R^{\mathrm{NH}}=\mathcal{L} \frac{\partial u^{L_{H}}}{\partial x} .
$$

We will show that, in geophysical mesoscale flows, $R^{\mathrm{NH}}$ can be an easy and useful correction, which allows one to recover part of the nonhydrostatic contribution $\delta \phi^{\mathrm{NH}}$ for a wide range of wavenumbers (i.e., scale of flows) when working with only hydrostatic variables.

\section{d. The nonlinear geopotential equation}

1) NONLINEAR OPERATOR WITH ZERO LARGE-SCALE FLOW, $U=0$

We define the nonlinear operator $\mathcal{N} \mathcal{L}$ :

$$
\begin{aligned}
\mathcal{N} \mathcal{L} & \equiv \partial_{t}+u \partial_{x}+w \partial_{z}+\lambda-K \nabla^{2} \\
& =\mathcal{L}+u \partial_{x}+w \partial_{z} .
\end{aligned}
$$

\section{2) NONLINEAR EQUATIONS}

The nonlinear and nonhydrostatic form of the equation is

$$
\begin{aligned}
\mathcal{N} \mathcal{L} u^{\mathrm{NL} L_{\mathrm{NH}}}-f v^{\mathrm{NL} L_{\mathrm{NH}}}+\phi_{x}^{\mathrm{NL}_{\mathrm{NH}}} & =0 ; \\
\mathcal{N} \mathcal{L} v^{\mathrm{NL}_{\mathrm{NH}}}+f u^{\mathrm{NL}_{\mathrm{NH}}} & =0 ; \\
\mathcal{N} \mathcal{L} w^{\mathrm{NL}_{\mathrm{NH}}}+\phi_{z}^{\mathrm{NL}_{\mathrm{NH}}} & =b^{\mathrm{NL}_{\mathrm{NH}}} ; \\
\mathcal{N} \mathcal{L} b^{\mathrm{NL}_{\mathrm{NH}}}+N^{2} w^{\mathrm{NL}_{\mathrm{NH}}} & =Q ; \\
u_{x}^{\mathrm{NL}}+w_{z}^{\mathrm{NL}} & =0 .
\end{aligned}
$$

The nonlinear and hydrostatic form is 


$$
\begin{aligned}
\mathcal{N} \mathcal{L} u^{\mathrm{NL}_{H}}-f \boldsymbol{v}^{\mathrm{NL}_{H}}+\phi_{x}^{\mathrm{NL}_{H}} & =0 ; \\
\mathcal{N} \mathcal{L} \boldsymbol{v}^{\mathrm{NL}_{H}}+f u^{\mathrm{NL}_{H}} & =0 ; \quad \phi_{z}^{\mathrm{NL}_{H}}=b^{\mathrm{NL}_{H}} ; \\
\mathcal{N} \mathcal{L} b^{\mathrm{NL}_{H}}+N^{2} w^{\mathrm{NL}_{H}} & =Q ; \\
u_{x}^{\mathrm{NL}_{H}}+w_{z}^{\mathrm{NL}_{H}} & =0 .
\end{aligned}
$$

\section{3) THE NONLINEAR GEOPOTENTIAL}

From Eq. (30), to derive the nonlinear geopotential, we differentiate by $x$ the first momentum equation and by $z$ the third momentum equation and add to obtain

$$
\begin{array}{r}
\nabla^{2} \phi^{\mathrm{NL}}=\frac{\partial b^{\mathrm{NL}}}{\partial z}-\left[\frac{\partial^{2}\left(u^{\mathrm{NL}}\right)^{2}}{\partial x^{2}}+2 \frac{\partial^{2}\left(w^{\mathrm{NL}} u^{\mathrm{NL}}\right)}{\partial x \partial z}\right. \\
\left.+\frac{\partial^{2}\left(w^{\mathrm{NL}}\right)^{2}}{\partial z^{2}}\right]+f \frac{\partial v^{\mathrm{NL}}}{\partial x} .
\end{array}
$$

\section{4) THE NONLINEAR CONTRIBUtion to THE GEOPOTENTIAL}

To derive the nonlinear contribution to the geopotential $\delta \phi^{\mathrm{NL}}$, from Eq. (30), we differentiate by $x$ the first momentum equation and multiply it by $\mathcal{N} \mathcal{L}$, add the second momentum equation differentiated by $x$ and multiplied by $f$, add the third momentum equation differentiated by $z$ and multiplied by $\mathcal{N} \mathcal{L}$, then add the buoyancy equation differentiated by $z$, yielding

$$
\begin{aligned}
\nabla^{2} \delta \phi^{\mathrm{NL}}= & -\left(\mathcal{N}(\mathcal{L})^{-1} \partial_{z}\left[u^{\mathrm{NL}} \partial_{x} b+w^{\mathrm{NL}} \partial_{z} b\right]\right. \\
& -\left[\left(\partial_{x} u^{\mathrm{NL}}\right)^{2}+2\left(\partial_{x} w^{\mathrm{NL}} \partial_{z} u^{\mathrm{NL}}\right)+\left(\partial_{z} w^{\mathrm{NL}}\right)^{2}\right] .
\end{aligned}
$$

In the previous form, the behavior of the solutions of the nonlinear partial differential equations (32) and (33) can be fully explored only using numerical techniques.

\section{e. Approximate nonlinear geopotential to the geopotential}

In order to analytically explore the behavior of the nonlinear geopotential in time-space, we use the following approximation:

$$
\begin{aligned}
\mathcal{L} \nabla^{2} \phi^{\mathrm{NL}} \approx & \mathcal{L} \nabla^{2} \phi^{L}-\partial_{z}\left[u^{L} \partial_{x} b+w^{L} \partial_{z} b\right] \\
& -\mathcal{L}\left[\left(\partial_{x} u^{L}\right)^{2}+2\left(\partial_{x} w^{L} \partial_{z} u^{L}\right)+\left(\partial_{z} w^{L}\right)^{2}\right] .
\end{aligned}
$$

The equation for the nonlinear contribution to the geopotential is then

$$
\begin{aligned}
\mathcal{L} & \nabla^{2}\left(\phi_{G}^{\mathrm{NL}}-\phi_{G}^{L}\right) \\
= & \mathcal{L} \nabla^{2} \delta \phi^{\mathrm{NL}} \\
\approx & -\partial_{z}\left[u^{L} \partial_{x} \Delta B+w^{L} \partial_{z} \Delta B\right] \\
& \quad-\mathcal{L}\left[\left(\partial_{x} u^{L}\right)^{2}+2\left(\partial_{x} w^{L} \partial_{z} u^{L}\right)+\left(\partial_{z} w^{L}\right)^{2}\right] .
\end{aligned}
$$

The buoyancy perturbation $\Delta B=-\left[\xi \partial_{x} B+\zeta \partial_{z} B\right]$ due to the air particle displacements is defined in Eq. (A27). This approximation holds whenever the nonlinear flow intensity is close to the linear flow intensity:

$$
\left|\frac{\mathbf{v}^{\mathrm{NL}}-\mathbf{v}^{L}}{\mathbf{v}^{\mathrm{NL}}}\right| \ll 1 \text {. }
$$

The two nonlinear contributions to the geopotential $\phi^{\mathrm{NL}}$ are

$$
\begin{aligned}
\mathcal{L} \nabla^{2} \phi_{B}^{\mathrm{NL}} & =-\partial_{z}\left[u^{L} \partial_{x} b+w^{L} \partial_{z} b\right] \\
\nabla^{2} \delta \phi_{D}^{\mathrm{NL}} & =\left[\left(\partial_{x} u^{L}\right)^{2}+2\left(\partial_{x} w^{L} \partial_{z} u^{L}\right)+\left(\partial_{z} w^{L}\right)^{2}\right] .
\end{aligned}
$$

$\delta \phi_{B}^{\mathrm{NL}}$ is due to the nonlinear advection of the buoyancy, such as the inland penetration of a sea-breeze front, which will be shown to be important at large time scales and large space lengths, while $\delta \phi_{D}^{\mathrm{NL}}$ is the dynamical pressure, whose relevance will be demonstrated at high pulsation rates and wavenumbers.

\section{Results and discussion}

In this section we examine the geopotential perturbations induced by a heat source in the planetary boundary layer, periodic in time and in the horizontal scale, which decays exponentially with altitude. We explore the dynamics of the mesoscale system with respect to the nonhydrostatic and hydrostatic, and nonlinear and linear approximations as a function of the horizontal scale and of the time scale in different environmental situations. We discuss the relative contributions of the different physical parameters such as the large-scale flow, atmospheric stability, dissipation, and diffusion. We show the validity of the different approximations in time-space diagrams.

In addition, we examine the dynamics and the pressure perturbations induced by an elevated and confined heat source due to the release of latent heat of condensation in a cloud.

\section{a. Periodic diabatic source in the $P B L$}

In nature, the intensity of the heat source in the PBL and its depth, $Q_{0}$ and $h_{0}$, have a large variability. However, throughout this section, we keep them constant to make the results more readable:

$$
\begin{aligned}
Q_{0} & =\omega_{E} h_{0} \frac{g \Theta_{z}}{\Theta}=\omega_{E} h_{0} N^{2} & \text { with } \omega_{E} & =\frac{2 \pi}{1 \text { day }} ; \\
h_{0} & =1 \mathrm{~km} \text { and } & h_{0} \Theta_{z} & =3^{\circ} .
\end{aligned}
$$

Then we study the mesoscale response as a function of the pulsation and wavenumbers of the forcing:

$$
Q_{a}(x, z, t)=Q_{0} \operatorname{expi}\left(\omega_{0} t+k_{0} x\right) \exp \left(-\mu_{0} z\right) .
$$

The wavenumbers and the pulsation rate are $\mu_{0}=1 / h_{0}$, $k_{0}=2 \pi / L$, and $\omega_{0}=2 \pi / T$, respectively, $T$ is the period of the forcing. 

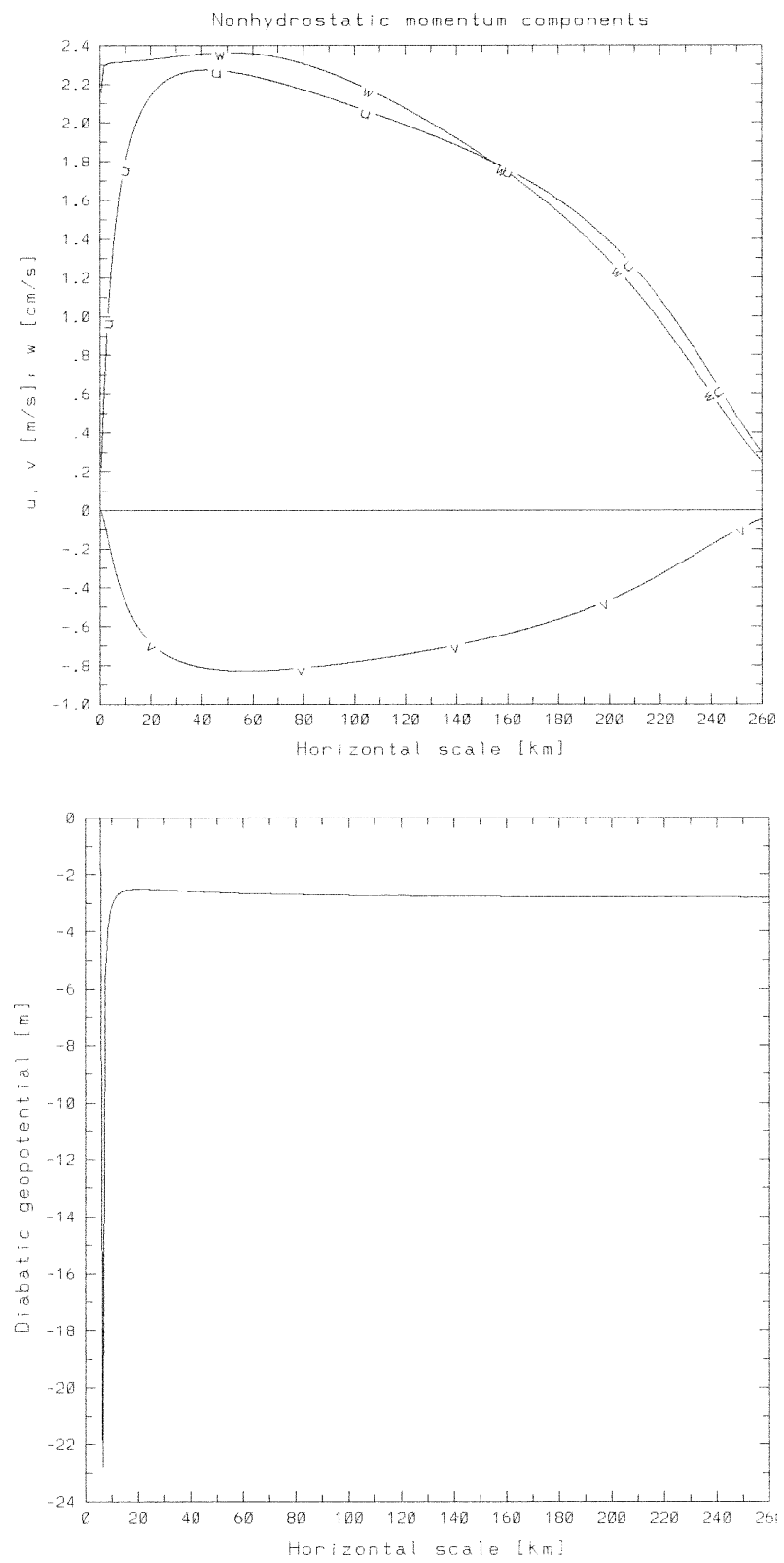

FIG. 1. Nonhydrostatic momentum $u^{\mathrm{NH}}\left(k_{0} x=-\pi / 2, z=0\right), v^{\mathrm{NH}}\left(k_{0} x\right.$ $=-\pi / 2, z=0) ; w^{\mathrm{NH}}\left(k_{0} x=0, z=h_{0}\right)$ and diabatic geopotential $\phi_{Q}\left(k_{0} x=0, z=0\right)$ as a function of the horizontal scale. The PBL forcing is periodic in time-space and exponentially decaying vertically, and the environmental parameters are $h_{0}=1 \mathrm{~km}, T=1$ day, $D^{-1}=2.5 \mathrm{~h}$ (and smaller at high wavenumbers), $f=10^{-4} \mathrm{~s}^{-1}$, and $N_{0}=10^{-2} \mathrm{~s}^{-1}$.

The amplitude of the nonhydrostatic momentum components and the diabatic geopotential as a function of the horizontal scale and in the absence of large-scale synoptic flow, are shown in Fig. 1. We show $u^{\mathrm{NH}}$ and $v^{\mathrm{NH}}$ at the surface $(z=0)$ and at a phase shift in the horizontal $\left(k_{0} x=-\pi / 2\right)$ from the location of the maximum of the warming. At the top of the CBL $\left(z=h_{0}\right)$ is $w^{\mathrm{NH}}$ and $\phi_{Q}$ is at the surface, while $w^{\mathrm{NH}}$ and $\phi_{Q}$ are both in phase with the peak of the heat source $Q$. The momentum amplitude increases as the horizontal scale increases up to the Rossby radius, and then decreases when the horizontal scale exceeds the Rossby radius, $L$ $\gg R_{0}$. At large horizontal scales, the flow intensity weakens since the momentum amplitudes are proportional to the horizontal buoyancy gradients $k_{0} Q$, which are small at large horizontal scales, $k_{0}=2 \pi / L$ [Eq. (A7)].

Since the forcing $Q$ is periodic in $x$ and decays exponentially in $z$ [Eq. (38)], the geopotential component directly induced by the heat source $\phi_{Q}$ is also periodic in $x$ and decays exponentially in $z$,

$$
\phi_{Q} \propto \exp i\left(\omega_{0} t+k_{0} x\right) \exp \left(-\mu_{0} z\right) .
$$

The symbol $\propto$ in Eq. (39) denotes directly proportional. The component $\phi_{Q}$ of the geopotential is linear.

To solve Eq. (19) for this component, we invert the operator $\nabla^{2} \equiv \partial_{x x}+\partial_{z z}$, which, when applied to $\phi_{Q}$, gives $\tilde{\nabla}^{-2}=1 /\left(\mu_{0}^{2}-k_{0}^{2}\right)$. It follows that this geopotential component reaches an asymptotic value for large horizontal scales, $L \gg R_{0}$, since in the Poisson equation, Eq. (19), $\tilde{\nabla}^{-2} \rightarrow 1 / \mu_{0}^{2}=$ constant, when $k_{0} \ll \mu_{0}\left(\mu_{0}=1 / h_{0}\right.$ and $\left.h_{0}=1 \mathrm{~km}\right)$. Note that $\phi_{Q}$ has a singularity when the horizontal wavenumber equals the vertical wavenumber, that is, when $k_{0} \rightarrow \mu_{0}$, then $\tilde{\nabla}^{-2}=1 /\left(\mu_{0}^{2}-k_{0}^{2}\right) \rightarrow \infty$ [Eq. (19)].

The Rossby radius concept adopted here has been extended to include pulsation rate and dissipation as in Dalu and Pielke (1993):

$$
R_{0}=h_{0} \sqrt{\frac{N^{2}+\mathcal{L}^{2}}{f^{2}+\mathcal{L}^{2}}} .
$$

The environmental parameters in Figs. $1-3$ are $f=$ $10^{-4} \mathrm{~s}^{-1}$ and $N_{0}=10^{-2} \mathrm{~s}^{-1}$. The time scale of the forcing $T$ is 1 day; the spindown time of the flow is $\operatorname{Re}\left\{\tilde{\mathcal{L}}^{-1}\right\}=\tau=\left[\lambda+K\left(k^{2}+\nu^{2}\right)\right]^{-1} \leq 2.5 \mathrm{~h}$. Flows with short wavelengths have a short lifetime, because diffusion activity increases with the square of the wavenumbers.

In Dalu et al. (1996) we showed that the intensity of a diabatically driven mesoscale flow generally decreases in the presence of large-scale synoptic flow. As the synoptic flow intensity increases, the flow transits from trapped to propagating waves. This transition occurs at lower synoptic winds when the horizontal wavelength of a heated patch of the PBL is smaller.

In the analytic model discussed in this paper, as also found in Dalu et al. (1996), the intensity generally decreases as the synoptic flow increases, where at shorter wavelengths the negative derivative is steeper. Interestingly, however, at the transition, when the flow switches abruptly from trapped to propagating waves, there is a sudden intensification of the updraft, which is steeper at shorter wavelengths. This intensification of the updraft, close to the transition zone from trapped to 

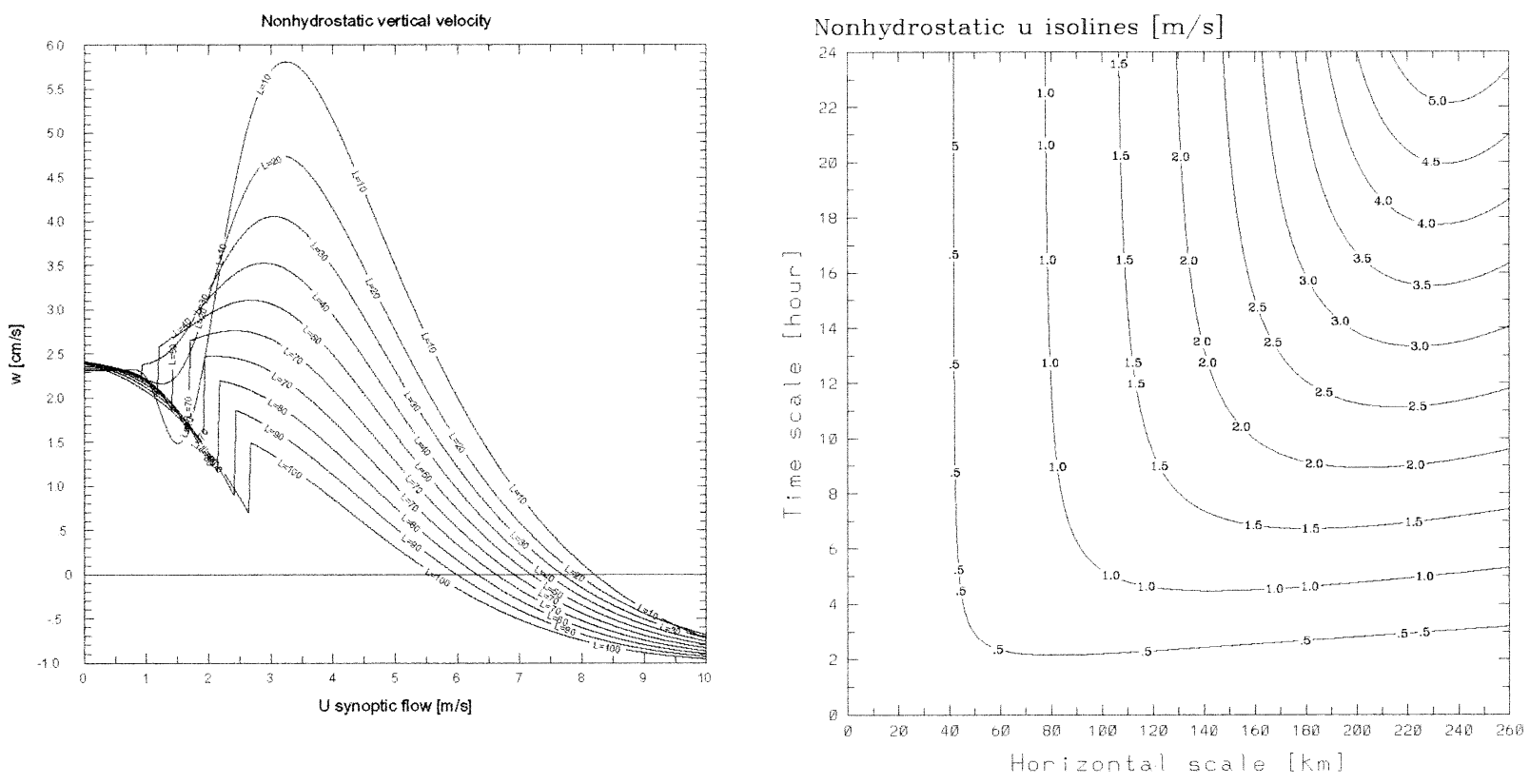

CONTOUR FROM O TO 6 BY .5

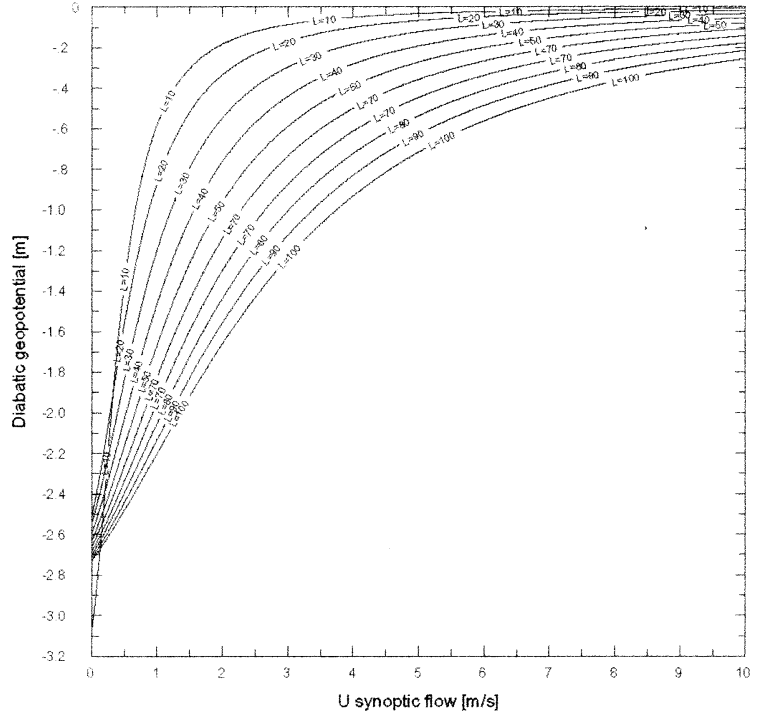

FIG. 2. Nonhydrostatic vertical momentum $w^{\mathrm{NH}}\left(k_{0} x=0, z=h_{0}\right)$, and diabatic geopotential $\phi_{Q}\left(k_{0} x=0, z=0\right)$, as a function of the synoptic wind intensity $U$ for horizontal wavelengths $L$, ranging from 10 to $100 \mathrm{~km}$, every $10 \mathrm{~km}$. Forcing and environmental parameters are as in Fig. 1.

propagating waves, can be very relevant in triggering cumulus convection at the top of the CBL.

Finally, when the synoptic flow is very strong, the mesoscale perturbation decays monotonically. Also the amplitude of $\phi_{Q}$ decays monotonically as the intensity

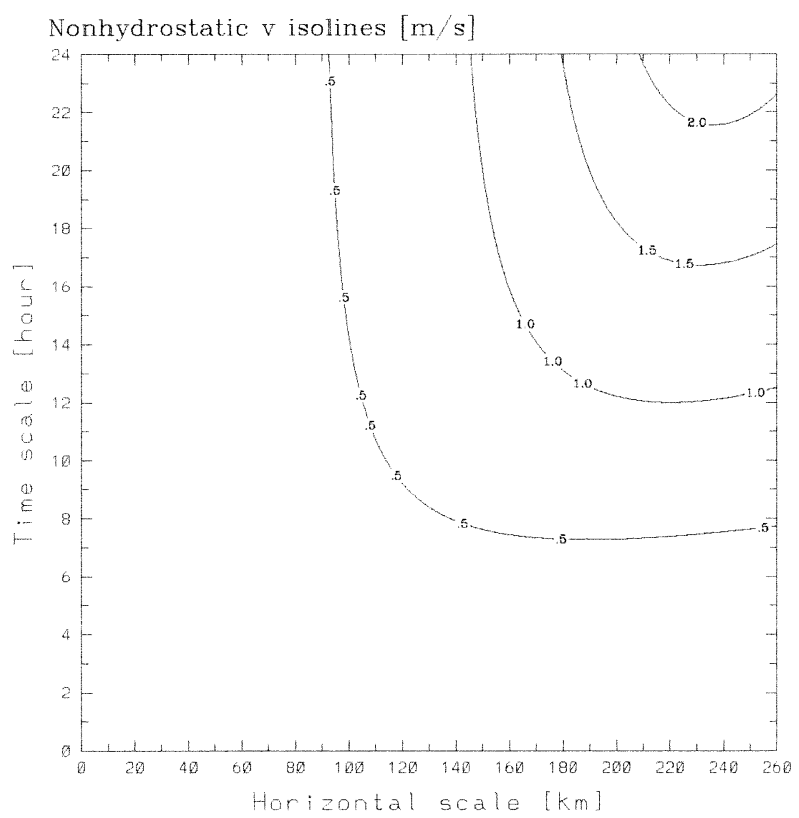

CONTOUR FROM O TO 6 BY .5

FIG. 3. Nonhydrostatic horizontal momentum components $u^{\mathrm{NH}}$ and $v^{\mathrm{NH}}$ as a function of time-space. Forcing and environmental parameters are as in Fig. 1.

of the large-scale flow increases. Values of $w^{\mathrm{NH}}$ at the top of the CBL and $\phi_{Q}$ at the surface as functions of the synoptic flow $U$ for horizontal scales ranging from $L=10-100 \mathrm{~km}$, at increments of $10 \mathrm{~km}$, are shown in Fig. 2.

Geophysical flows are mainly characterized by their time and space scales; therefore, we need to explore 

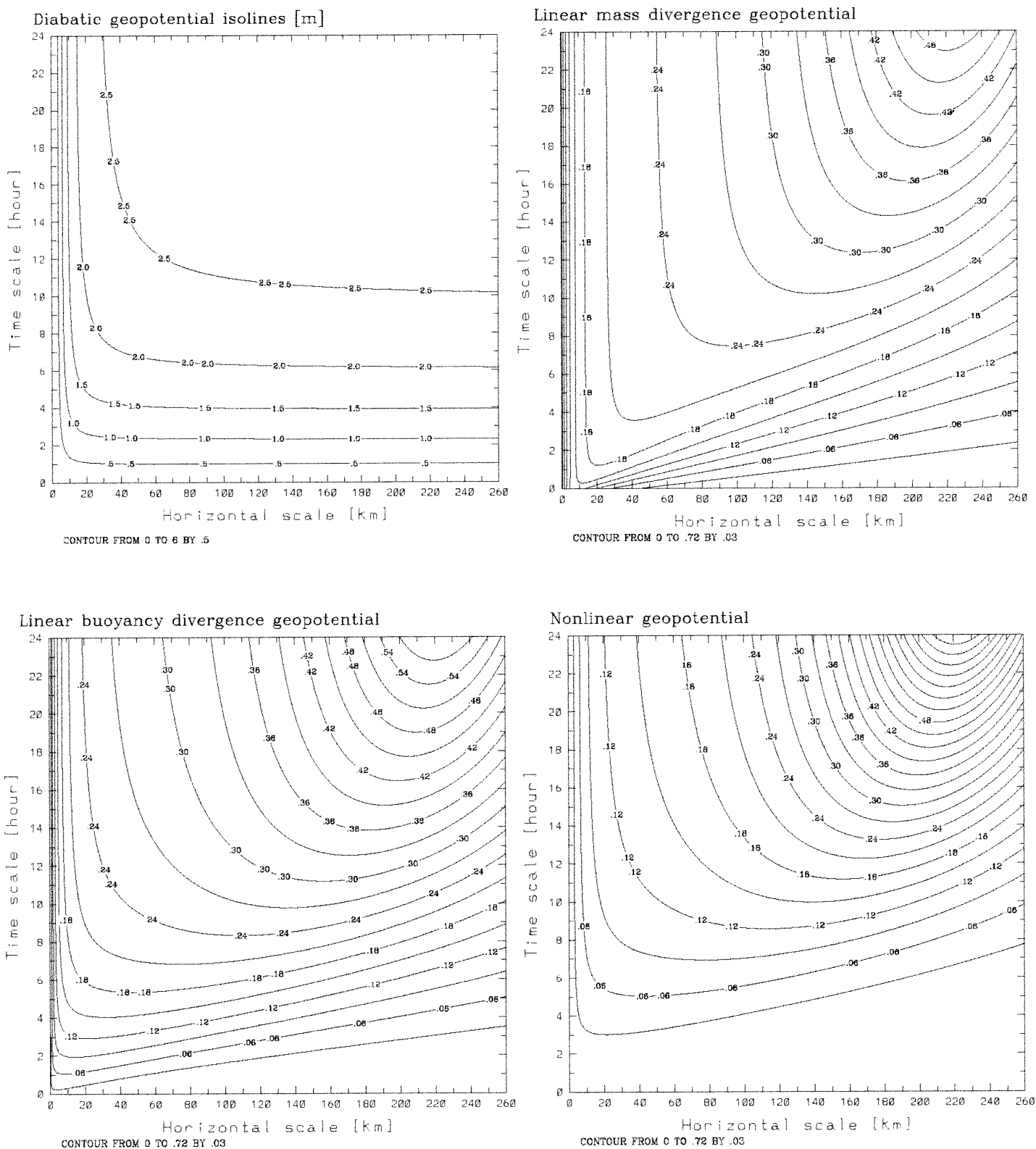

FIG. 4. Geopotentials as a function of time-space: $\phi_{Q}(\mathrm{~m}) ; \phi_{D}, \phi_{B}$, and $\delta \phi^{\mathrm{NL}}=\left(\delta \phi_{B}^{\mathrm{NL}}+\delta \phi_{D}^{\mathrm{NL}}\right)$ are normalized by $\phi_{Q}$. Forcing and environmental parameters are as in Fig. 1.

the dynamics of the geopotentials in a wide region of time-space. The time scale for mesoscale atmospheric flows ranges from a few minutes for a CBL thermal to 1 day for a sea breeze, while the space scale ranges from hundreds of meters to hundreds of kilometers. Figure 3 shows that the amplitude of $u^{\mathrm{NH}}=\mu_{0} \psi$ (A6) grows in the time-space diagram, and that the amplitude of the across-front momentum component $v^{\mathrm{NH}}=$ $-\mathcal{L}^{-1} f u^{\mathrm{NH}}(\mathrm{A} 26)$ has a slower growth in time-space than $u^{\mathrm{NH}}$. Note that $u^{\mathrm{NH}}$ lags the source $Q$, and $v^{\mathrm{NH}}$ lags $u^{\mathrm{NH}}$. Only the nonhydrostatic momentum components are shown, since the difference between the nonhy- drostatic dynamics and the hydrostatic dynamics is relevant only in a close neighborhood where $k_{0} \approx \mu_{0}$ and $\omega_{0}^{2}-D^{2} \approx N^{2}$.

In Fig. 4 we investigate the geopotentials in the time-space diagram. We show the geopotential $\phi_{Q}$, and the isobaric anomaly in meters as directly deformed by the heat source. The other geopotentials are normalized by $\phi_{Q}$. The geopotential $\phi_{D}$ results from the convergence-divergence of mass, and is always a relevant term in ageostrophic geophysical mesoscale stratified flows. An important fraction of $\phi_{O}$ is $\phi_{D}$. Since $\phi_{D} \propto\left(N^{2}-f^{2}\right) k_{0} u^{\mathrm{NH}}$, this geopotential 
becomes generally small when the atmosphere is nonstratified $N^{2} \approx 0$, with only a contribution due to the remaining inertia force, which cannot be neglected in semigeostrophic flows such as cold fronts, or in the regional lifting by convergence lines (Crook and Klemp 2000).

The geopotential that results from the convergencedivergence of the linear diabatic buoyancy perturbation $\phi_{B}$ is also an important fraction of $\phi_{Q}$, even if $\phi_{D}$ is usually more effective than $\phi_{B}$ at all wavelengths. The nonlinear advection of buoyancy accounts for most of the nonlinear contribution to the geopotential, $\delta \phi^{\mathrm{NL}}=$ $\left(\delta \phi_{B}^{\mathrm{NL}}+\delta \phi_{D}^{\mathrm{NL}}\right)$. The nonlinear advection of buoyancy $\delta \phi_{B}^{\mathrm{NL}}$ is especially important at large time-space scales, where the dynamical pressure is usually negligible; $\delta \phi_{D}^{\mathrm{NL}} \ll \delta \phi_{B}^{\mathrm{NL}}$ when $T \approx 1$ day and $L \approx R_{0}$, as occurs in a sea-breeze front penetrating inland. The importance of $\delta \phi_{D}^{\mathrm{NL}}$ is usually limited to the regions of the atmosphere where there are very rapid variations of velocities in time-space.

The nonhydrostatic contribution and the nonhydrostatic residual are very close almost everywhere in the time-space diagram, $\delta \phi^{\mathrm{NH}} \approx R^{\mathrm{NH}}$. In the regions of the atmosphere where the pulsation rate and the wavenumbers are high, the difference between the nonhydrostatic and the hydrostatic dynamics is large; in the CBL this is the domain of the thermals. To further explain this concept, in the next section we extend our study to a developing cloud. The more complex cloud dynamics clearly show where in the atmosphere the nonhydrostatic effect matters.

\section{b. Cumulus cloud dynamics and geopotentials}

In this section, we examine the dynamics and the geopotentials generated by an elevated confined diabatic source as would occur in a cloud. We analyze the dynamics generated by a diabatic heat $Q$ and how the mesoscale dynamics deforms the geopotentials. The source is confined to a horizontal scale, $-L<x<L$, and a vertical scale, $h_{0}<z<h_{1}$.

$$
\begin{aligned}
Q_{b}(x, z, t)= & Q_{0} \sin \left(\omega_{0} t\right)\left[\operatorname{He}\left(h_{1}-z\right)-\operatorname{He}\left(h_{0}-z\right)\right] \\
& \times[\operatorname{He}(L+x)-\operatorname{He}(L-x)],
\end{aligned}
$$

where

$$
Q_{0}=\omega_{0} N^{2}\left(h_{1}-h_{0}\right)=\omega_{0}\left(h_{1}-h_{0}\right) \frac{g \Theta_{z}}{\Theta} .
$$

the Heaviside step function (He) is $\mathrm{He}(\mathrm{x})=1$ for $x \geq$ 0 and $\mathrm{He}(\mathrm{x})=0$ for $x<0$. Here: $Q \neq 0$ when $-L<$ $x<L$ and $h_{0}<z<h_{1}$.

In order to study the behavior of the flow in the cloud, where the flow in the cloud is nonlinear and nonhydrostatic, and where the linear and/or hydrostatic approximations can be valid, we chose a heat source with an aspect ratio of order one and a time scale of $1 \mathrm{~h}$ :

$$
\left(h_{1}-h_{0}\right) \approx 2 L \text { and } \omega_{0}=\frac{2 \pi}{1 \mathrm{~h}} .
$$

\section{1) LARGE CUMULUS}

Figure 5 depicts the dynamics and the pressure field of a large cumulus with a $3-\mathrm{km}$ base, $3-\mathrm{km}$ radius, and $3-\mathrm{km}$ depth. The geopotential induced by the diabatic source $\phi_{Q}$, in meters, is shown at the peak of the release of the latent heat. Here $Q$ generates a pressure dipole with a high pressure pole aloft and a low pressure pole below the cloud down to the surface. The nonhydrostatic streamfunction $\psi^{\mathrm{NH}}\left(\mathrm{km} \times \mathrm{m} \mathrm{s}^{-1}\right)$ shows the low-level convergence and the upper-level divergence. In response to the pressure force $\nabla \phi_{Q}$, the airflow converges toward the low pressure pole at low levels, which reduces in size and partially fills in, while the upper-level high pressure pole weakens and reduces in size because of airflow divergence. The nonlinear contribution to the geopotential $\delta \phi^{\mathrm{NL}}$ is about $15 \%$ of the total pressure field $\phi^{\mathrm{NL}}$. While the linear residuals are about 1/5 of the nonlinear residual, they are, however, still sizeable. Comparison between the nonhydrostatic contribution $\delta \phi^{\mathrm{NH}}$, and the nonhydrostatic residual $R^{\mathrm{NH}}$ shows that the flow in the cloud is nonhydrostatic where the updraft is more intense, and that the Song et al. (1985) correction captures most of the residual except in these limited regions of very strong updraft.

\section{2) Midsize AND SMALl CUMUlus}

Figure 6 shows the dynamics and pressure field in a midsize cumulus cloud with its base at $1 \mathrm{~km}$, and with a $2-\mathrm{km}$ radius and depth. Figure 7 depicts the dynamics and pressure field in a low, small cumulus cloud with its base at $500 \mathrm{~m}$, and with a $1-\mathrm{km}$ radius and depth.

The updraft is less intense in smaller clouds with limited vertical development. The nonhydrostatic residual correction works better and the dynamic pressure is more likely to be negligible. In deep clouds, the updraft is more intense with strong gradients and variability, which makes the nonhydrostatic residual correction not workable and the dynamics pressure nonnegligible.

\section{Further simple useful results}

We analyzed how mesoscale dynamics modifies the pressure field in response to a diabatic source. The geopotential has one linear term generated by the diabatic source, plus four additional terms that are dynamically induced: two terms are linear and two terms are nonlinear. Each of these four terms are generally nonhydrostatic. However, in a large region of the atmosphere, the hydrostatic approximation can be made, or most of the nonhydrostatic residual can be recovered 
(a) Diabatic geopotential isolines [m]

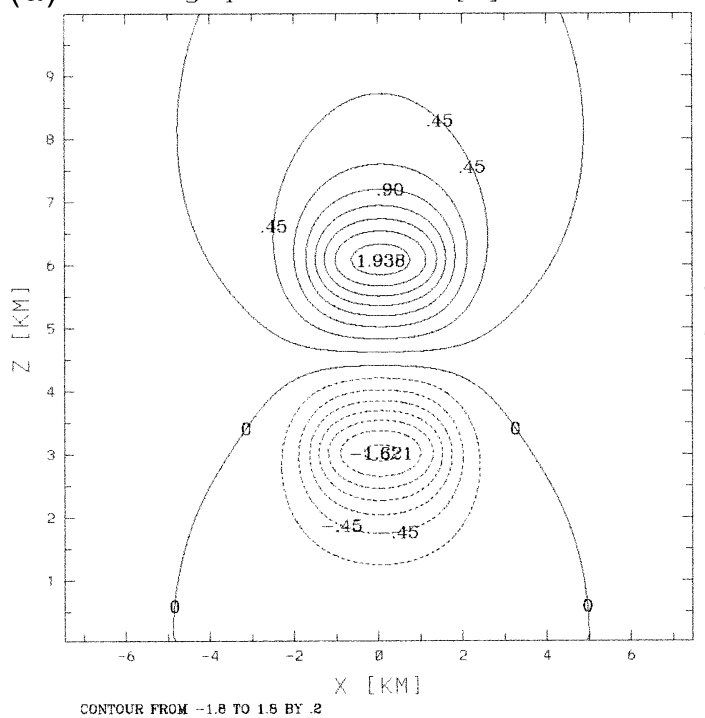

Nonhydrostratic streamfunction $[\mathrm{km} \mathrm{x} \mathrm{m/s}]$

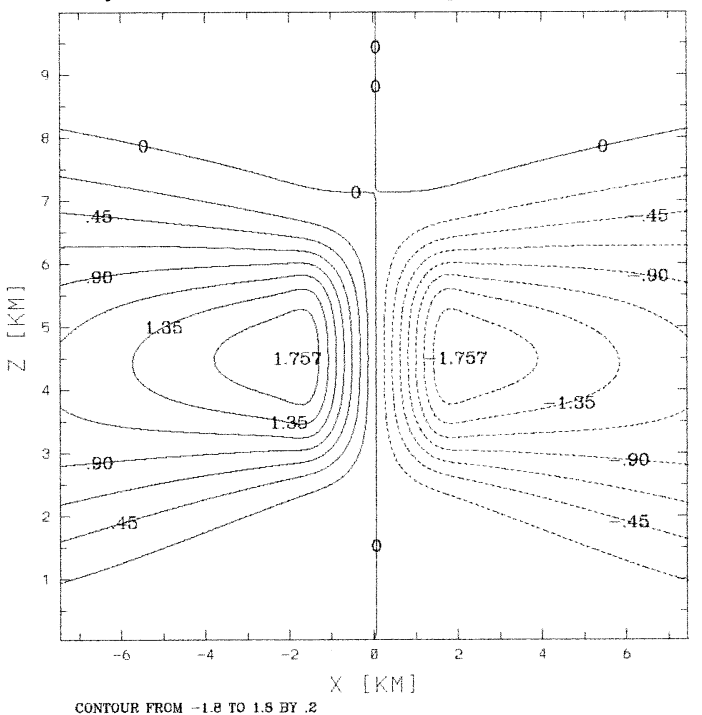

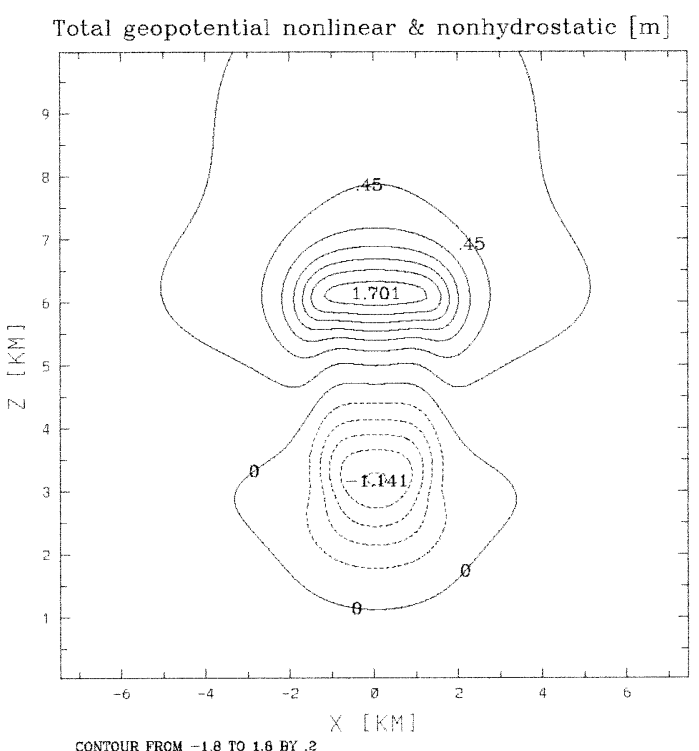

FIG. 5. Large cumulus dynamics, geopotential, and geopotential residual: diabatic geopotential $\phi_{Q}$, nonhydrostatic streamfunction $\psi$, and nonhydrostatic and nonlinear total pressure field $\phi_{G}$. Nonlinear contribution $\delta \phi^{\mathrm{NL}}=\left(\delta \phi_{B}^{\mathrm{NL}}+\right.$ $\left.\delta \phi_{D}^{\mathrm{NL}}\right)$, nonhydrostatic contribution $\delta \phi^{\mathrm{NH}}=\left(\phi^{\mathrm{NH}}-\phi^{\mathrm{H}}\right)$, and nonhydrostatic residual $R^{\mathrm{NH}}$.

using the Song et al. (1985) correction. In this section, we revisit the nonhydrostatic versus the hydrostatic residual, analyze the four mesoscale geopotential terms, and determine where and when they are important in order to facilitate a first-order quantitative evaluation.

\section{a. Hydrostatic approximation}

It is well known that flow is nonhydrostatic when the aspect ratio is of order one. In addition, we found that the time scale is also an important parameter. From the appendix Eqs. (A16)-(A18), we can derive the relative amplitude of the flow and the relative phase lag:

$$
\begin{aligned}
& 1-\left(\frac{\psi_{0}^{\mathrm{NH}}}{\psi_{0}^{H}}\right)^{2} \approx\left(D^{2}-\omega_{0}^{2}\right) k^{2}\left[\frac{\psi_{0_{1}}^{\mathrm{NH}}+\psi_{0_{1}}^{H}}{k_{0} Q_{0}}\right] \text { and } \\
& {\left[1-\frac{\delta^{L_{H}}}{\delta^{L_{\mathrm{NH}}}}\right] \approx\left[1-\frac{\psi_{0_{1}}^{\mathrm{NH}}}{\psi_{0_{1}}^{H}}\right] .}
\end{aligned}
$$

When the pulsation rate $\omega_{0}$ is larger than dissipation rate $D$, the hydrostatic flow is more intense and is also quicker in responding to the forcing (Fig. 8). The role 

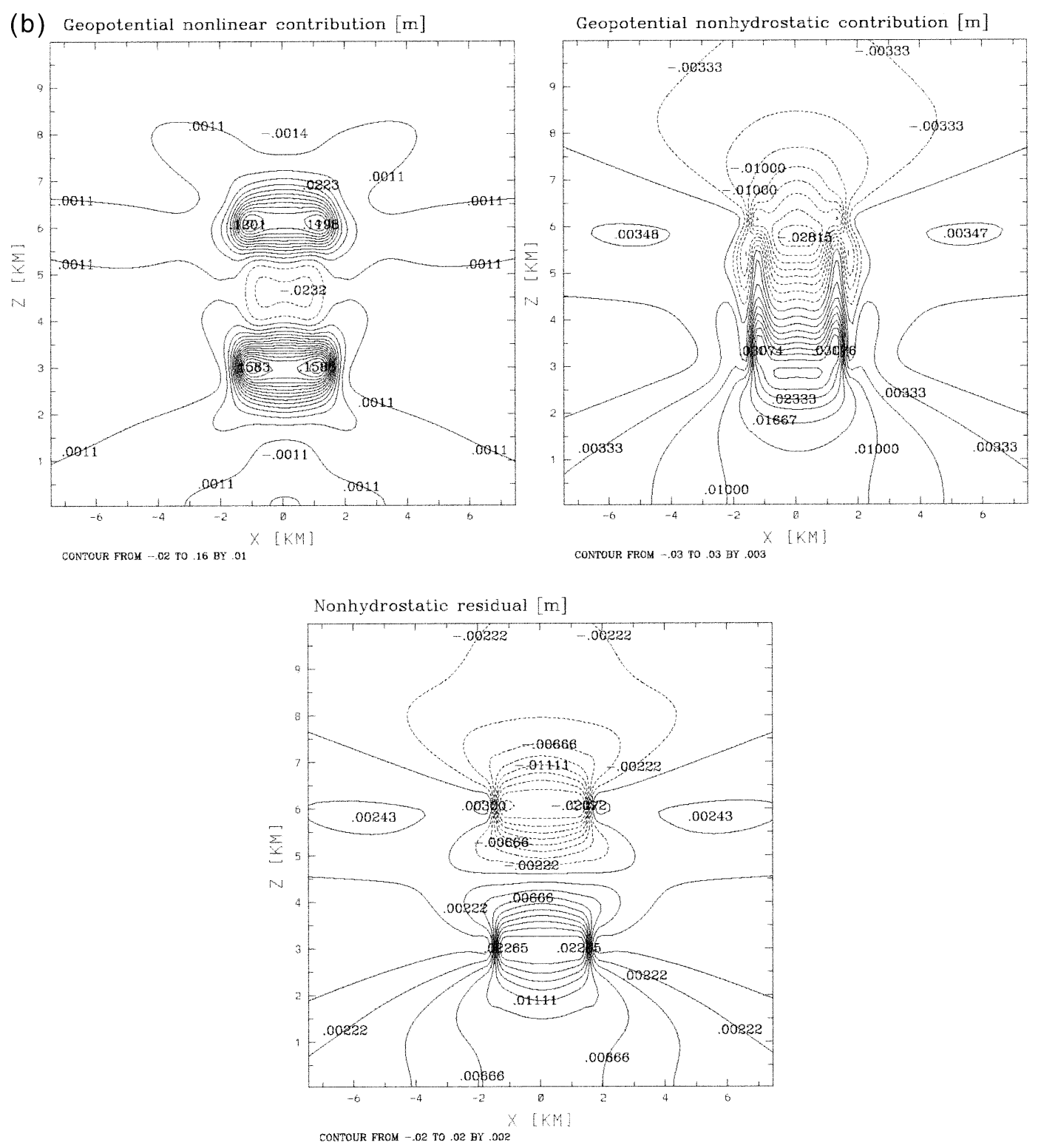

FIG. 5. (Continued)

of the Brunt-Väisälä frequency is more evident when $\omega_{0}^{2} \gg D^{2}=\left[\lambda+K\left(k^{2}+\mu^{2}\right)\right]^{2}:$

$$
\begin{aligned}
{\left[1-\frac{\psi_{0}^{H}}{\psi_{0}^{\mathrm{NH}}}\right] } & =\frac{\omega_{0}^{2} k^{2}}{\left(f^{2}-\omega_{0}^{2}\right) \mu^{2}+N^{2} k^{2}} \\
& \approx \frac{\omega_{0}^{2} k^{2}}{N^{2} k^{2}-\omega_{0}^{2} \mu^{2}} .
\end{aligned}
$$

Since $f^{2} \ll N^{2}$, the flow is highly nonhydrostatic when $k^{2} \approx \mu^{2}$ and $\omega_{0}^{2} \approx N^{2}$. Therefore, a large difference in relative amplitude between the nonhydrostatic and the hydrostatic flow occurs when the aspect ratio is of order one and the pulsation rate is comparable to the Brunt-Väisälä frequency. In the simulations performed by Cassano and Parish (2000) on an Antarctic katabatic flow, it is clearly shown that the difference between the nonhydrostatic and the hydrostatic flow is large and confined to the region of the atmosphere where the aspect ratio is of order one.

\section{b. Importance of the linear mesoscale geopotential terms}

The geopotential due to the divergence-convergence $\phi_{D}$ is important in the stably stratified region of the 
atmosphere, and when $f^{2} \ll N^{2}$ its relative importance is

$$
\frac{\phi_{Q}-\phi_{D}}{\phi_{Q}} \approx 1-\frac{N^{2} \partial_{x} u}{\partial_{z} Q} \approx 1-\frac{k u}{\omega} .
$$

Note that $\phi_{D}$ is important when the horizontal convergence-divergence is comparable with the vertical divergence of diabatic buoyancy within the same column of air. When $Q_{0} \propto \omega h_{0} N^{2}$ as in a periodic PBL [Eq. (A10)], $\phi_{D}$ is important when $k u \approx \omega$; the advection time has to be comparable to the pulsation period of the forcing.

The geopotential induced by the divergence-convergence of diabatic buoyancy, $\phi_{B}$ is

$$
\begin{aligned}
\left|\frac{\phi_{Q}-\phi_{B}}{\phi_{Q}}\right| & \approx 1-\left|\frac{\left(u \partial_{x} B+w \partial_{z} B\right)}{Q}\right| \\
& \approx 1-\left|\frac{(k u-\mu w)}{\omega}\right| .
\end{aligned}
$$

In a periodic PBL, $B=\omega^{-1} Q$ [Eq. (5)], then $\phi_{B}$ is important when $\omega \approx(k u+\mu w)$. The advection time has to be comparable to the pulsation period; therefore, $\phi_{D}$ and $\phi_{B}$ are usually equally important.

Since the mesoscale flow is mainly driven by the pressure gradient forces directly generated by the diabatic heat source $\boldsymbol{\nabla} \phi_{D}$ in order to weigh the relative importance of the pressure terms $\phi_{D}, \phi_{B}$, and $\delta \phi_{B}^{\mathrm{NL}}$, we show the threshold at which these terms exceed $10 \%, 25 \%$, and $50 \%$ of $\phi_{Q}$ as a function of the time and space scales (Fig. 9). When a pressure term exceeds $10 \%$ of $\phi_{D}$, we assume the term cannot be neglected. When it exceeds the $25 \%$ threshold, it produces a clearly sizeable deformation of the pressure pattern and of the flow field. Finally, where the pressure terms exceed the $50 \%$ threshold, it means that the mesoscale dynamics at that spatial scale has sufficient time to be fully developed.

\section{c. Importance of the nonlinear mesoscale geopotential terms}

Let us quantify the relative importance of the nonlinear geopotential term versus the linear term:

$$
\frac{\phi_{D}-\delta \phi_{D}^{\mathrm{NL}}}{\phi_{D}} \approx \frac{\mathcal{L}^{-1}\left(N^{2}-f^{2}\right) \partial_{x} u-\left[\left(\partial_{x} u^{L}\right)^{2}+2 \partial_{x} w^{L} \partial_{z} u^{L}+\left(\partial_{z} w^{L}\right)^{2}\right]}{\mathcal{L}^{-1}\left(N^{2}-f^{2}\right) \partial_{x} u} \approx 1-2\left|\frac{\mathcal{L}(k u+\mu w)}{N^{2}}\right| .
$$

The nonlinear dynamical pressure contribution $\delta \phi_{D}^{\mathrm{NH}}$ is important when the advection time multiplied by the period of the pulsation rate is comparable to the BruntVäisälä period squared, $2|\mathcal{L}(k u+\mu w)| \approx N^{2}$. Therefore, the pulsation and wavenumber need to be very high. Here $\delta \phi_{D}^{\mathrm{NL}}$ is relevant in the regions of rapidly changing gradients and pulsation rate (Fig. 8). The nonlinear buoyancy advection contribution $\delta \phi_{B}^{\mathrm{NL}}$ needs longer time scales, of the order of 1 day, to establish itself (Fig. 9):

$$
\begin{aligned}
& \frac{\phi_{B}-\delta \phi_{B}^{\mathrm{NL}}}{\phi_{B}} \\
& \approx \frac{\left(u \partial_{x} B+w \partial_{z} B\right)-\left(u \partial_{x} \Delta B+w \partial_{z} \Delta B\right)}{\left(u \partial_{x} B+w \partial_{z} B\right)} \\
& \approx 1-\left|\frac{(k u+\mu w)}{\mathcal{L}}\right|
\end{aligned}
$$

Since the amplitude of $\Delta B \propto\left|\mathcal{L}^{-1}(k u+\mu w) B\right|$ [Eq. (A27)], when we compare $\delta \phi_{B}^{\mathrm{NL}}$ to $\phi_{B}$, the nonlinear advection of buoyancy contribution is important when $\tilde{\mathcal{L}} \approx(k u+\mu w)$. Or, when we compare $\delta \phi_{B}^{\mathrm{NL}}$ to $\phi_{Q}$, the nonlinear advection of the buoyancy term is important when $(\omega \tilde{\mathcal{L}}) \approx(k u+\mu w)^{2}$. In this situation the advection time squared has to be comparable to the period of the pulsation squared. The threshold at which $\delta \phi_{B}^{\mathrm{NL}}$ exceeds $10 \%, 25 \%$, and $50 \%$ of $\phi_{Q}$ is shown in Fig. 9.

\section{Conclusions}

The mesoscale flow field is driven by the pressure gradient force generated by the diabatic source. Four additional pressure gradient terms are generated by the mesoscale dynamics: two terms are linear and two terms are nonlinear; these terms are generally nonhydrostatic. Depending on the space scales and time scales of the mesoscale system, these terms can be negligible, or contribute significantly to the mesoscale dynamics.

It is important to determine when the flow is predominantly hydrostatic. For these situations, for example, remotely sensed monitoring of the vertical and horizontal fields of temperature can be used to uniquely describe the spatial variation of the pressure field. When the flow is predominantly linear, exact analytic solution techniques can be used, rather than relying on approximated numerical solution methodologies that introduce computational error, as shown in Pielke (2002).

As we demonstrate in the paper, in a large range of time and space scales of the atmospheric flow, the hy- 


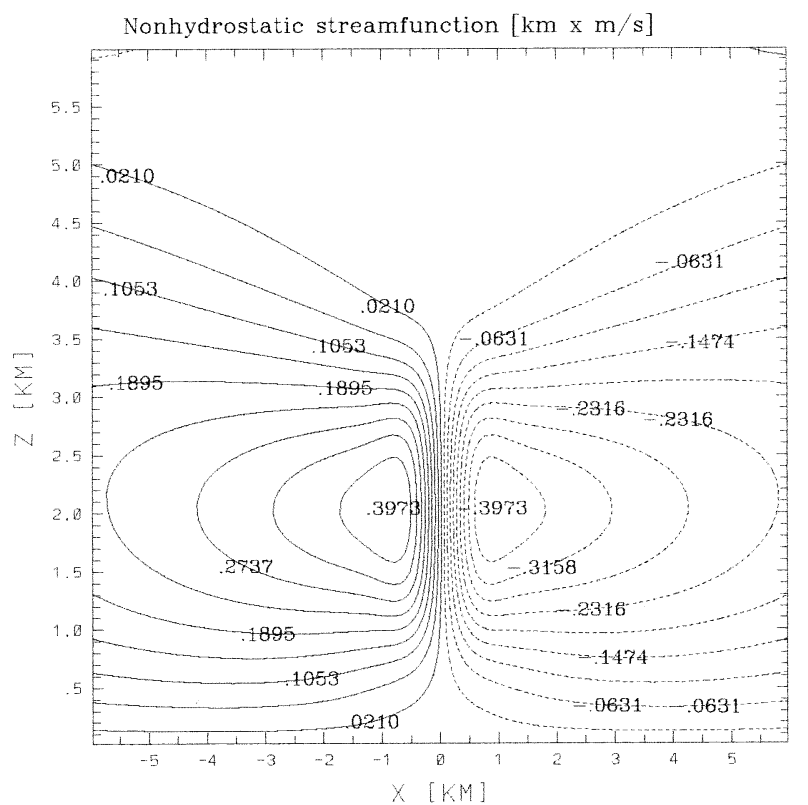

CONTOUR FROM -.4 TO .4 BY .04

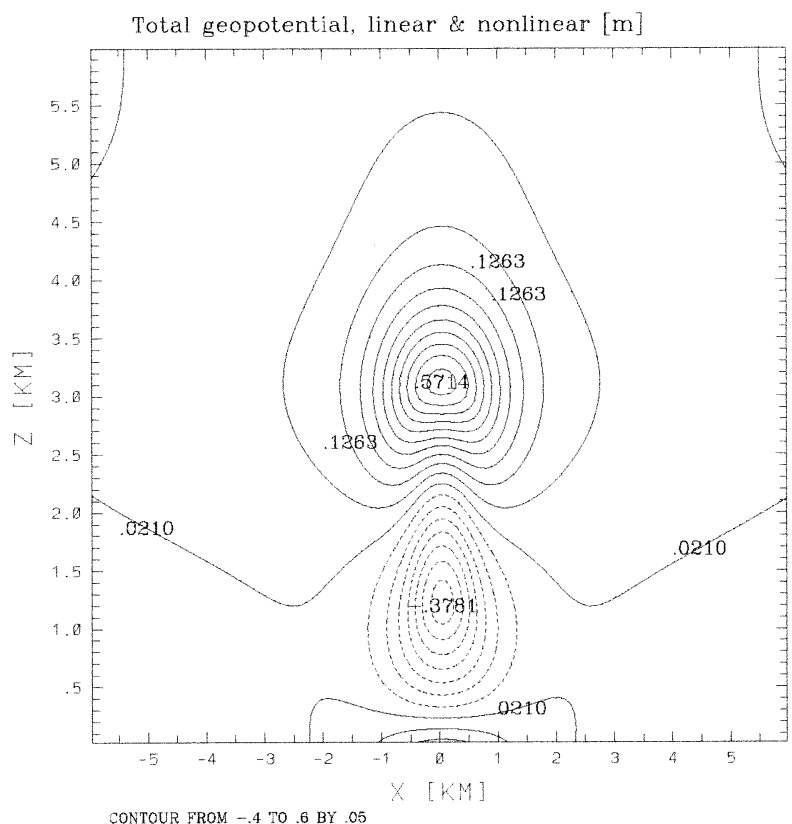

FIG. 6. Midsize cumulus dynamics and geopotential: nonhydrostatic streamfunction $\psi$, and nonhydrostatic and nonlinear total pressure field $\phi_{G}$.

drostatic approximation can be made. Most of the nonhydrostatic residual can be recovered using the correction that we suggest, which fails, however, when the aspect ratio is about 1 and the pulsation is comparable with the Brunt-Väisälä frequency; in this region the nonlinear dynamical pressure is also nonnegligible.

The two linear pressure gradient terms associated with convergence-divergence of mass and with con-
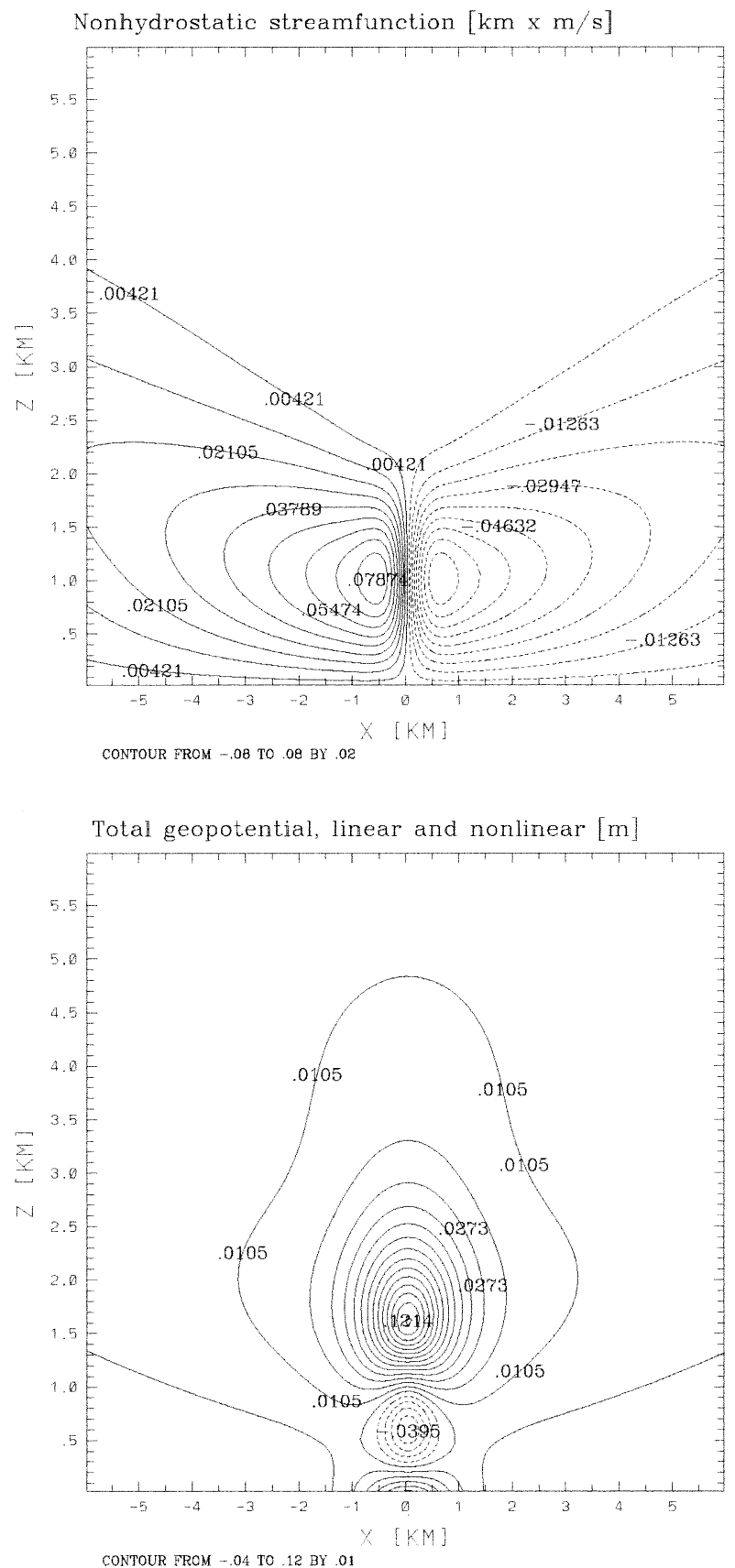

FIG. 7. Small cumulus dynamics and geopotential: nonhydrostatic streamfunction $\psi$, and nonhydrostatic and nonlinear total pressure field $\phi_{G}$.

vergence-divergence of buoyancy are not usually negligible in atmospheric flows. The nonlinear pressure gradient term generated by the nonlinear advection of buoyancy cannot be neglected when the time scale of the flow is comparable to the inertial period. While the nonlinear dynamical pressure is relevant in the regions of rapidly changing gradients and pulsation rate.

We conclude that, in mesoscale flows, the pressure 

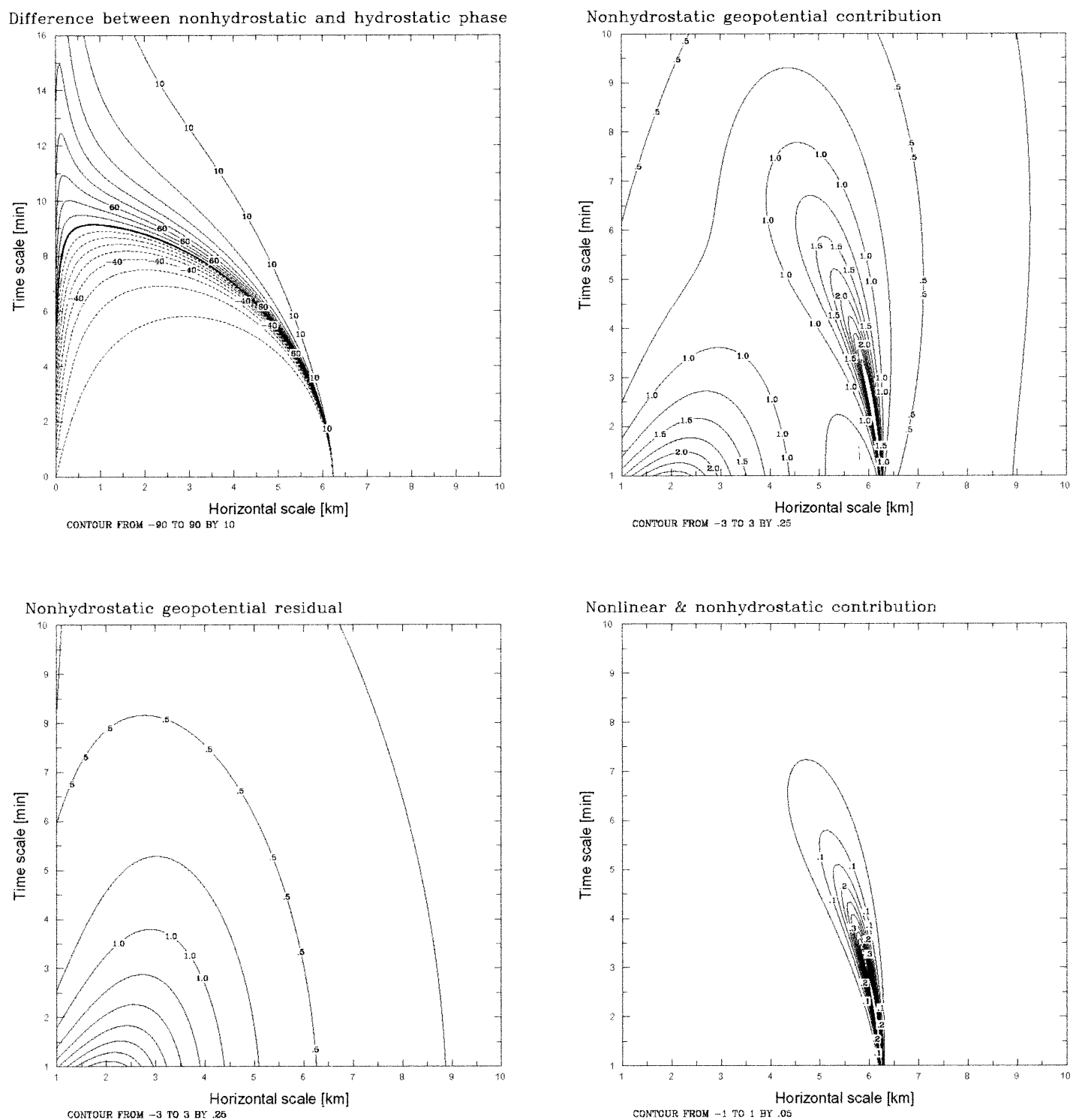

FIG. 8. Phase lag difference, $\delta_{w^{N H}}-\delta_{w^{H}}$, between the nonhydrostatic and the hydrostatic updraft. The nonhydrostatic contribution to the geopotential $\delta \phi_{N} H$, the nonhydrostatic geopotential residual $R_{N} H$, and the nonlinear contribution to the geopotential $\delta \phi_{N} L$ are normalized by $\phi_{Q}$.

gradients are linear, except when the time scale is on the order of the Brunt-Väisälä period. In this case, the flow is also highly nonhydrostatic. When the time scale is on the order of the inertial period, the nonlinear advection of temperature becomes important, although the flow is hydrostatic. It would be useful to merge our deterministic approach with the stochastic approach of Wang et al. (1996) to further investigate this region of rapidly changing gradients and pulsation.

The approach presented is an extension and a generalization of the theory reported by Weidman and Pielke (1983) and Song et al. (1985), using the theoretical model developed by Dalu and Pielke (1989). An extension of our study, presently two-dimensional, to a fully three-dimensional flow would allow the analysis of other important phenomena as, for instance, the asymmetric growth of a cyclonic-anticyclonic dipole in a veering vertically sheared flow (Rotunno and Klemp 1982).

Acknowledgments. Support was provided by the National Science Foundation under Grant ATM-9910857 and U.S. Department of Defense DAAD19-01-2-0018. G. A. Dalu and M. Baldi also acknowledge the support of the Italian Space Agency (MeditRain - ASI) and the Italian CNR Short-Term Mobility Program. Dallas Staley and Tara Green very capably handled the final preparation of the paper. 

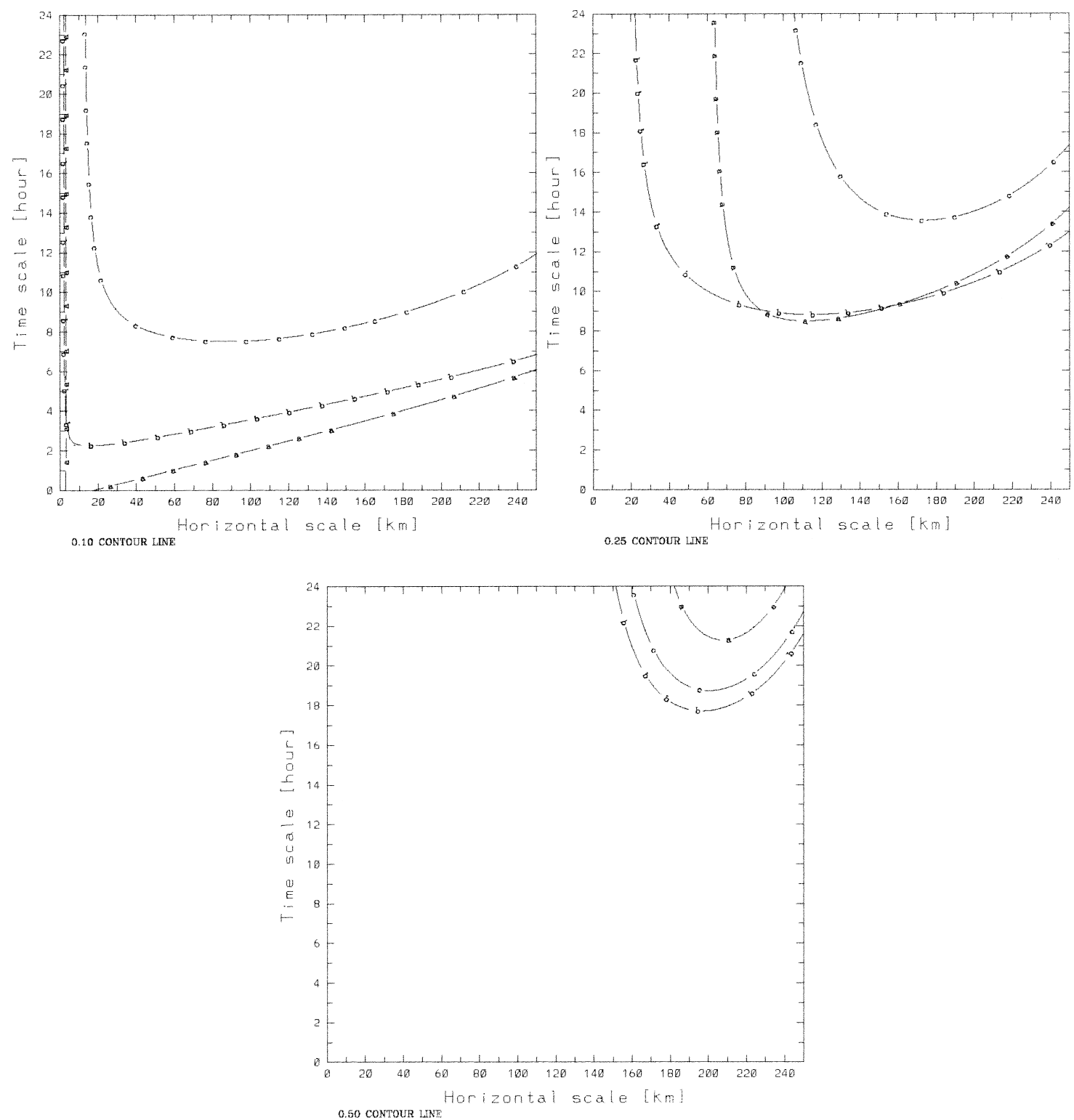

FIG. 9. Regions in time-space where $\phi_{D}, \phi_{B}$, and $\delta \phi_{B}^{\mathrm{NL}}$ exceed $\phi_{Q}$ by $10 \%, 25 \%$, and $50 \%$.

\section{APPENDIX}

\section{Model Derivation}

a. The governing primitive equations

The two-dimensional and Boussinesq primitive equations are

$$
\begin{array}{r}
\left(\frac{\partial}{\partial t}+\lambda\right) u+U \frac{\partial u}{\partial x}-f v+\frac{\partial \phi}{\partial x}=K\left(\frac{\partial^{2}}{\partial x^{2}}+\frac{\partial^{2}}{\partial z^{2}}\right) u \\
\left(\frac{\partial}{\partial t}+\lambda\right) v+U \frac{\partial v}{\partial x}+f u=K\left(\frac{\partial^{2}}{\partial x^{2}}+\frac{\partial^{2}}{\partial z^{2}}\right) v
\end{array}
$$

$$
\left(\frac{\partial}{\partial t}+\lambda\right) w+U \frac{\partial w}{\partial x}+\frac{\partial \phi}{\partial z}-b=K\left(\frac{\partial^{2}}{\partial x^{2}}+\frac{\partial^{2}}{\partial z^{2}}\right) w
$$

$$
\begin{aligned}
\left(\frac{\partial}{\partial t}+\lambda\right) b+U \frac{\partial b}{\partial x}+N^{2} w & =Q+K\left(\frac{\partial^{2}}{\partial x^{2}}+\frac{\partial^{2}}{\partial z^{2}}\right) b \\
\frac{\partial u}{\partial x}+\frac{\partial w}{\partial z} & =0 .
\end{aligned}
$$

The flow is driven by the horizontal gradient of the diabatic source $Q$ in Eq. (A4). The geopotential $\phi$ is defined as $g \delta h$, where $\delta h$ is the vertical displacement from a barotropic reference surface. The geopotential 
and pressure gradient forces are equivalent, as shown in Eq. (1).

\section{b. The streamfunction equation}

Using the mass continuity equation (A5), we define a streamfunction $\psi$ :

$$
\frac{\partial \psi}{\partial z}=u \text { and } \quad \frac{\partial \psi}{\partial x}=-w .
$$

The equation for the streamfunction $\psi$ is derived from Eqs. (A1)-(A5):

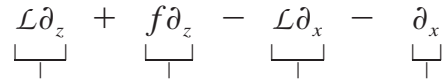

(Eq. A1) (Eq. A2) (Eq. A3) (Eq. A4)

$$
\Rightarrow \underbrace{\left(\mathcal{L}^{2}+f^{2}\right) \frac{\partial^{2} \psi^{L_{\mathrm{NH}}}}{\partial z^{2}}+\left(\mathcal{L}^{2}+N^{2}\right) \frac{\partial^{2} \psi^{L_{\mathrm{NH}}}}{\partial x^{2}}=-\frac{\partial Q}{\partial x},}_{\text {(Eq. A7) }}
$$

and its hydrostatic approximation is

$$
\left(\mathcal{L}^{2}+f^{2}\right) \frac{\partial^{2} \psi^{L_{H}}}{\partial z^{2}}+N^{2} \frac{\partial^{2} \psi^{L_{H}}}{\partial x^{2}}=-\frac{\partial Q}{\partial x} .
$$

\section{c. Dynamics in a periodic PBL}

\section{1) DiABATIC FORCING}

$$
\begin{aligned}
Q_{a}(x, z, t) & =Q_{0} \exp \left(-\mu_{0} z\right) \operatorname{Re}\left[\exp i\left(\omega_{0} t+k_{0} x\right)\right] \\
& =Q_{0} \exp \left(-\mu_{0} z\right) \cos \left(\omega_{0} t+k_{0} x\right)
\end{aligned}
$$

where

$$
\begin{aligned}
\omega_{E} Q_{0} & =N^{2} h_{0}=g \frac{\Theta_{z} h_{0}}{\Theta}, \quad k_{0}=\frac{2 \pi}{L}, \text { and } \\
\mu_{0} & =\frac{1}{h_{0}} .
\end{aligned}
$$

The diabatic forcing intensity is $Q_{0}, k_{0}$ is the horizontal, $\mu_{0}$ is the vertical wavenumber, $L$ is the horizontal wavelength, and $h_{0}$ is the depth of the CBL.

\section{2) NONHYDROSTATIC COEFFICIENTS}

In the following equations, when the flow is nonhydrostatic, $a, b$, and $c$ have the following values:

$$
\begin{array}{ll}
a=f^{2}-\Omega^{2}+D^{2}, \quad b=N^{2}-\Omega^{2}+D^{2}, & \text { and } \\
c=2 D \Omega . & \text { (A11) }
\end{array}
$$

\section{3) Hydrostatic COEFFICIENTS}

$a=f^{2}-\Omega^{2}+D^{2}, \quad b=N^{2}, \quad$ and $c=2 D \Omega$.

\section{4) FoRCED MODES}

The dynamical response to the diabatic forcing in Eq. (A9) is of the form

$$
\begin{aligned}
\psi_{\text {forced }}(x, z, t) & \\
=[ & \psi_{0_{1}} \sin \left(\omega_{0} t+k_{0} x\right) \\
& \left.+\psi_{0_{2}} \cos \left(\omega_{0} t+k_{0} x\right)\right] \exp \left(-\mu_{0} z\right) .
\end{aligned}
$$

From the streamfunction equation, Eq. (A7), the amplitudes $\psi_{0_{1}}$ and $\psi_{0_{2}}$ are computed by separately equating the sines and the cosines:

$\left(a \mu_{0}^{2}-b k_{0}^{2}\right) \psi_{0_{1}}-c\left(\mu_{0}^{2}-k_{0}^{2}\right) \psi_{0_{2}}=k_{0} Q_{0}$

$c\left(\mu_{0}^{2}-k_{0}^{2}\right) \psi_{0_{1}}+\left(a \mu_{0}^{2}-b k_{0}^{2}\right) \psi_{0_{2}}=0$,

from which the in-phase and the out-of-phase forced responses are, respectively,

$\psi_{0_{1}}=k_{0} Q_{0} \frac{\left(a \mu_{0}^{2}-b k_{0}^{2}\right)}{\left(a \mu_{0}^{2}-b k_{0}^{2}\right)^{2}+c^{2}\left(\mu_{0}^{2}-k_{0}^{2}\right)^{2}}$

$\psi_{0_{2}}=-k_{0} Q_{0} \frac{c\left(\mu_{0}^{2}-k_{0}^{2}\right)}{\left(a \mu_{0}^{2}-b k_{0}^{2}\right)^{2}+c^{2}\left(\mu_{0}^{2}-k_{0}^{2}\right)^{2}}$.

The flow amplitude $\psi_{0}$ and its phase shift $\delta$ are

$$
\begin{aligned}
\psi_{0} & =\left(\psi_{0_{1}}^{2}+\psi_{0_{2}}^{2}\right)^{1 / 2} \\
& =\frac{k_{0} Q_{0}}{\left[\left(a \mu_{0}^{2}-b k_{0}^{2}\right)^{2}+c^{2}\left(\mu_{0}^{2}-k_{0}^{2}\right)^{2}\right]^{1 / 2}} \\
\delta & =\tan ^{-1}\left(\frac{\psi_{0_{2}}}{\psi_{0_{1}}}\right) .
\end{aligned}
$$

The forced mode is

$$
\psi_{\text {forced }}(x, z, t)=\psi_{0} \sin \left(\omega_{0} t+k_{0} x+\delta\right) \exp \left(-\mu_{0} z\right) .
$$

\section{5) FREE MODES}

The vertical wavenumber of the free mode $\mu_{1}$ is computed from the streamfunction equation Eq. (A7) in the absence of forcing,

$$
\begin{gathered}
\psi_{\text {free }} \propto \exp i\left(\omega_{0} t+k_{0} x\right) \exp \left(-\mu_{1} z\right), \\
{\left[(a+i c) \mu_{1}^{2}-(b+i c) k_{0}^{2}\right] \psi_{\text {free }}=0,} \\
\mu_{1}^{2}=k_{0}^{2}\left(\frac{b+i c}{a+i c}\right) \\
\mu_{1}= \pm k_{0}\left(\frac{b^{2}+c^{2}}{a^{2}+c^{2}}\right)^{1 / 4} \exp \left(i \frac{\beta}{2}\right), \\
\beta=\tan ^{-1}\left(\frac{c}{b}\right)-\tan ^{-1}\left(\frac{c}{a}\right) .
\end{gathered}
$$

Physical constraints define the sign of the vertical wavenumber: the energy has to be finite and propagate down- 
stream (Smith 1989). The free mode is a trapped wave when $\mu_{1}=\mu_{1}$. It is a propagating wave when $\mu_{1}=$ $\mu_{1}$, and it is a propagating decaying wave when $\mu_{1}=$ $\mu_{1_{r}}+i \mu_{1_{i}}$.

$$
\begin{aligned}
& \psi_{\text {free }}(x, z, t) \\
& \quad=\psi_{0} \sin \left(\omega_{0} t+k_{0} x+\mu_{1_{i}} z+\delta\right) \exp \left(-\mu_{1_{r}} z\right)
\end{aligned}
$$

Trapped free modes are confined within the the PBL near the ground while the free modes with complex vertical wavenumbers propagate into the midtroposphere. The free mode $\psi$ has the same amplitude of the forced mode $\psi_{0}$ and it is subtracted from the forced modes to match the condition of vanishing vertical flow at the ground:

$$
\begin{aligned}
\psi(x, z, t)= & \psi_{\text {forced }}(x, z, t)-\psi_{\text {free }}(x, z, t), \text { with } \\
\psi(x, z=0, t)=0 & \\
\psi(x, z, t)=\psi_{0} & \sin \left(\omega_{0} t+k_{0} x+\delta\right) \exp \left(-\mu_{0} z\right) \\
& \quad-\sin \left(\omega_{0} t+k_{0} x+\mu_{1_{i}} z+\delta\right) \\
& \left.\times \exp \left(-\mu_{1_{r}} z\right)\right] .
\end{aligned}
$$

\section{6) Momentum COMPONENTS}

The horizontal and vertical momentum components $u$ and $w$ are

$$
\begin{aligned}
& u(x, z, t) \\
& =\psi_{0}\left[\mu_{1_{r}} \sin \left(\omega_{0} t+k_{0} x+\mu_{1_{i}} z+\delta\right)\right. \\
& \times \exp \left(-\mu_{1_{r}} z\right)-\mu_{1_{i}} \cos \left(\omega_{0} t+k_{0} x+\mu_{1_{i}} z+\delta\right) \\
& \times \exp \left(-\mu_{1_{r}} z\right)-\mu_{0} \sin \left(\omega_{0} t+k_{0} x+\delta\right) \\
& \left.\times \exp \left(-\mu_{0} z\right)\right]
\end{aligned}
$$

$w(x, z, t)$

$$
\begin{gathered}
=\psi_{0}\left[k_{0} \cos \left(\omega_{0} t+k_{0} x+\mu_{1_{i}} z+\delta\right) \exp \left(-\mu_{1_{r}} z\right)\right. \\
\left.-k_{0} \cos \left(\omega_{0} t+k_{0} x+\delta\right) \exp \left(-\mu_{0} z\right)\right] .
\end{gathered}
$$

The along- $x$ axis $\xi$ and along- $z$ axis $\zeta$ particle displacements are

$$
\begin{aligned}
& \xi(x, z, t)=\int u(x, z, t) d t=\mathcal{L}^{-1} u \text { and } \\
& \zeta(x, z, t)=\int w(x, z, t) d t=\mathcal{L}^{-1} w .
\end{aligned}
$$

The along-y axis velocity component $v$ and the along$y$ axis particle displacement $\eta$ are

$$
\begin{aligned}
& v=-f \int u(x, z, t) d t=-f \xi \text { and } \\
& \eta=\int v(x, z, t) d t=-f \int \xi d t .
\end{aligned}
$$

The rate at which the buoyancy is perturbed by the advection through the diabatically perturbed region $B$ in Eq. (5), and the buoyancy perturbation $\Delta B$ due to the displacement of the air particles through the same region are, respectively,

$$
\begin{aligned}
& -\left(u^{L_{\mathrm{NH}}} \partial_{x} B+w^{L_{\mathrm{NH}}} \partial_{z} B\right) \quad \text { and } \\
\Delta B= & -\left[\xi \partial_{x} B+\zeta \partial_{z} B\right] .
\end{aligned}
$$

\section{d. Dynamics induced by a confined heat source}

\section{1) DiABATIC FORCING}

The Fourier transformation of an elevated and confined heat source, Eq. (41), is

$$
\begin{aligned}
\tilde{Q}_{b}\left(k, \nu, \omega_{0}\right)= & \int_{-\infty}^{\infty} d t \int_{0}^{\infty} d x \int_{0}^{\infty} d z \\
& \times Q_{b}(x, z, t) \sin (\nu z) \cos (k x) \operatorname{expi}\left(\omega_{0} t\right) \\
= & Q_{0}\left[\frac{\sin (k L)}{k}\right]\left[\frac{\cos \left(\nu h_{0}\right)-\cos \left(\nu h_{1}\right)}{\nu}\right] \\
& \times \frac{1}{2 i}\left[\delta\left(\omega-\omega_{0}\right)-\delta\left(\omega+\omega_{0}\right)\right]
\end{aligned}
$$

$\tilde{Q}_{b}\left(k, \nu, \omega_{0}\right)=Q_{0} \tilde{r}(k, \nu)$

$$
\times \frac{1}{2 i}\left[\delta\left(\omega-\omega_{0}\right)-\delta\left(\omega+\omega_{0}\right)\right] .
$$

In the previous equation, $\delta\left(\omega-\omega_{0}\right)$ is the Dirac function centered at $\omega_{0}$ (pulsation of the forcing) and $k$ and $\nu$ are the horizontal and the vertical wavenumbers. The Fourier transform is even along $x$, and it is odd along $z$ in order to match the lower boundary condition of vanishing flow, $\psi(x, z=0, t)=0$.

\section{2) StREAMFUnCTION} is

The Fourier transform of the streamfunction Eq. (A7)

$$
\nu^{2}\left(\tilde{\mathcal{L}}^{2}+f^{2}\right) \tilde{\psi}+k^{2}\left(\tilde{\mathcal{L}}^{2}+N^{2}\right) \tilde{\psi}=-k \tilde{Q}_{b}\left(k, \nu, \omega_{0}\right)
$$

$$
\tilde{\psi}\left(k, \nu, \omega_{0}\right)=\frac{-k \tilde{Q}_{b}\left(k, \nu, \omega_{0}\right)}{\nu^{2}\left(\tilde{L}^{2}+f^{2}\right)+k^{2}\left(\tilde{\mathcal{L}}^{2}+N^{2}\right)} .
$$

Then, the streamfunction $\psi$ is computed through the inverse transform of Eq. (A29):

$\psi(x, z, t)=\varphi_{1}(x, z) \sin \left(\omega_{0} t\right)+\varphi_{2}(x, z) \cos \left(\omega_{0} t\right)$,

where $\varphi_{1}(x, z)$ is the component in phase with the forcing and $\varphi_{2}(x, z)$ is the out of phase component. Here, $\varphi_{1}(x, z)$ and $\varphi_{2}(x, z)$ are the Fourier inverse transforms of $\tilde{\varphi}_{1}(k, \nu)$ and $\tilde{\varphi}_{2}(k, \nu)$, respectively:

$$
\begin{aligned}
& \tilde{\varphi}_{1}(k, \nu) \\
& \quad=-k Q_{0} \tilde{r}(k, \nu) \frac{a \nu^{2}+b k^{2}}{\left(a \nu^{2}+b k^{2}\right)^{2}+c^{2}\left(k^{2}+\nu^{2}\right)^{2}}
\end{aligned}
$$




$$
\begin{aligned}
& \tilde{\varphi}_{2}(k, \nu) \\
& \quad=k Q_{0} \tilde{r}(k, \nu) \frac{c\left(k^{2}+\nu^{2}\right)}{\left(a \nu^{2}+b k^{2}\right)^{2}+c^{2}\left(k^{2}+\nu^{2}\right)^{2}} .
\end{aligned}
$$

\section{3) NONHYdRostatic COEFFICIENTS}

$$
\begin{aligned}
a & =D^{2}-\omega_{0}^{2}+f^{2} ; & b & =D^{2}-\omega_{0}^{2}+N_{0}^{2} ; \\
c & =2 \omega_{0} D ; & D & =\lambda+K\left(k^{2}+\nu^{2}\right) .
\end{aligned}
$$

\section{4) Hydrostatic COEFFicients}

$$
\begin{aligned}
a & =D^{2}-\omega_{0}^{2}+f^{2} ; & b & =N_{0}^{2} ; \\
c & =2 \omega_{0} D ; & D & =\lambda+K\left(k^{2}+\nu^{2}\right) .
\end{aligned}
$$

\section{REFERENCES}

Cassano, J. L., and T. R. Parish, 2000: An analysis of the nonhydrostatic dynamics in numerically simulated Antarctic katabatic flows. J. Atmos. Sci., 57, 891-898.

Cotton, W. R., and R. A. Anthes, 1989: Storm and Cloud Dynamics. Academic Press, $883 \mathrm{pp}$

Crook, N. A., and J. B. Klemp, 2000: Lifting by convergence lines. J. Atmos. Sci., 57, 873-890.

Dalu, G. A., and R. A. Pielke, 1989: An analytical study of the sea breeze. J. Atmos. Sci., 46, 1815-1825.

$\ldots$, and —_, 1993: Vertical heat fluxes generated by mesoscale atmospheric flow induced by thermal inhomogeneities in the PBL. J. Atmos. Sci., 50, 919-926.

_ _ _ M. Baldi, and X. Zeng, 1996: Heat and momentum fluxes induced by thermal inhomogeneities. J. Atmos. Sci., 53, 32863302 .

-, P. L. Vidale, and M. Baldi, 2000: Heat transport and weakening of the atmospheric stability induced by mesoscale flows. J. Geophys. Res., 105 (D7), 9349-9363.

LaPage, W. R., 1961: Complex Variables and the Laplace Transform for Engineers. Dover, $475 \mathrm{pp}$.

Molemaker, M. J., and H. Dijkstra, 2000: Stability of a cold core eddy in the presence of convection: Hydrostatic versus nonhydrostatic modeling. J. Phys. Oceanogr., 30, 475-494.

Pielke, Sr., R. A., 2002: Mesoscale Meteorological Modeling. 2d ed. Academic Press, 676 pp.

Rõõm, R., and A. Männik, 1999: Responses of different nonhydrostatic, pressure-coordinate models to orographic forcing. J. Atmos. Sci., 56, 2553-2570.

Rotunno, R., 1983: On the linear theory of land and sea breeze. $J$. Atmos. Sci., 40, 1999-2009.

— and J. Klemp, 1982: The influence of the shear-induced pressure gradient on thunderstorm motion. Mon. Wea. Rev., 110, 136151.

Seman, C. J., 1994: A numerical study on nonlinear nonhydrostatic conditional symmetric instability in a convectively unstable atmosphere. J. Atmos. Sci., 51, 1352-1371.

Smith, R. B., 1989: Hydrostatic air-flow over mountains. Advances in Geophysics, Vol. 31, Academic Press, 1-41.

Song, J. L., R. A. Pielke, R. W. Arritt, and R. C. Kessler, 1985: A method to determine nonhydrostatic effects within subdomains in a mesoscale model. J. Atmos. Sci., 42, 2110-2119.

Stull, R. B., 1988: An Introduction to Boundary Layer Meteorology. Kluwer Academic, $666 \mathrm{pp}$.

Sun, W.-Y., 1984: Numerical analysis for hydrostatic and nonhydrostatic equations of inertial-internal gravity waves. Mon. Wea. Rev., 112, 259-268

Thunis, P., and A. Clappier, 2000: Formulation and evaluation of a nonhydrostatic mesoscale vorticity model (TVM). Mon. Wea Rev., 128, 3236-3251.

Wang, J., R. L. Bras, and E. A. B. Eltahir, 1996: A stochastic linear theory of mesoscale circulation induced by the thermal heterogeneity of the land surface. J. Atmos. Sci., 53, 3349-3366.

Weidman, S. T., and R. A. Pielke, 1983: A more accurate method for the numerical solution of nonlinear partial differential equations. J. Comput. Phys., 49, 342-348. 